\title{
Simulating air entrainment and vortex dynamics in a hydraulic jump
}

\author{
Adam Witt ${ }^{1 *}$, John Gulliver ${ }^{1}$, Lian Shen ${ }^{2}$ \\ ${ }^{1}$ St. Anthony Falls Laboratory, Department of Civil Engineering, University of Minnesota, 2 Third Ave \\ SE, Minneapolis, MN 55414, USA. \\ ${ }^{2}$ St. Anthony Falls Laboratory, Department of Mechanical Engineering, University of Minnesota, 2 Third \\ Ave SE, Minneapolis, MN 55414, USA. \\ *Corresponding author: Tel: 001763443 9221; E-mail address: witt0225@umn.edu
}

\begin{abstract}
The air entrainment characteristics of three separate Froude number hydraulic jumps are investigated numerically using an unsteady RANS, realizable $k$ - $\varepsilon$ turbulence model, with a Volume of Fluid treatment for the free surface. Mean velocity profiles, average void fraction, and Sauter mean diameter compare favorably with experimental data reported in literature. In all simulations, time-averaged void fraction profiles show good agreement with experimental values in the turbulent shear layer and an accurate representation of interfacial aeration at the free surface. Sauter mean diameter is well represented in the shear layer, and free surface entrainment results indicate bubble size remains relatively unchanged throughout the depth of the jump. Several different grid resolutions are tested in the simulations. Significant improvements in void fraction and bubble size comparison are seen when the diameter to grid size ratio of the largest bubbles in the shear layer surpasses eight. A three-dimensional simulation is carried out for one Froude number jump, showing an improvement in the prediction of entrained air and bubble size compared with two-dimensional results at a substantial increase in computation time. An analysis of three-dimensional vorticity shows a complex interaction between spanwise and streamwise vortical structures and entrained air bubbles. The jump is similar to a turbulent mixing layer, constrained by the free surface, with vortex pairing and subsequent fluctuations in free surface elevation. Downstream fluctuations of the toe are associated with a roll up of the primary spanwise vortex, fluctuations of the free surface, and counter-rotating streamwise vortex pairs. The action of these flow structures is likely responsible for the improvement in three-dimensional results.
\end{abstract}

Keywords: Air concentration, air entrainment, bubble dynamics, hydraulic jump, void fraction, vortex 


\section{Introduction}

A hydraulic jump arises when supercritical flow transitions to subcritical flow, resulting in turbulence, energy dissipation, air entrainment, and higher rates of gas transfer. A hydraulic jump is frequently employed downstream of dams to dissipate energy and improve oxygen levels in the flowing water. Air entrainment occurs when pockets of atmospheric gases are captured as bubbles during an oscillation of the toe of the jump. These pockets of air experience breakup in the turbulent shear region, producing bubbles that either rise into the roller where they are released to the atmosphere, or travel downstream with the bulk flow where air-liquid gas transfer can occur. Aeration at the free surface is simultaneously enhanced by contributions from breaking waves.

The bubbly flow structure of an air entraining hydraulic jump has been studied in many experimental investigations (Rajaratnam, 1967; Resch et al., 1974; Avery and Novak, 1978; Murzyn and Chanson, 2007). Impingement of the recirculating roller upon the upstream jet creates the primary source of air bubbles transported downstream. The void fraction, $\varphi$, or the volume proportion of air to water and air together, is often used to characterize the time-averaged behavior of bubbles within the jump. The frequency of bubbles and the maximum void fraction are strongly linked to the inflow Froude number (Chanson and Brattberg, 2000), defined as $F r=U_{o} /(g d)^{0.5}$, where $U_{o}$ and $d$ are respectively velocity and depth of the upstream jet, and $g$ is the gravitational acceleration. Void fraction measurements have been carried out with relative accuracy using dual-tip conductivity probes (Straub and Anderson, 1958; Rajaratnam, 1967; Chanson, 2007b), hot-wire anemometry (Resch et al., 1974), visual techniques (Hoyt and Sellin, 1989; Mossa and Tolve, 1998), and optical fiber probes (Murzyn et al., 2005). It has been found that the maximum void fraction at a given elevation increases rapidly downstream from the toe, peaking in the roller region before decreasing gradually downstream (Rajaratnam, 1962). The shape of the void fraction profile within the hydraulic jump varies depending on inflow conditions and the boundary layer thickness of the inflow (Resch et al., 1974). Herein, the discussion will focus exclusively on hydraulic jumps with a thin boundary layer in the incident flow, otherwise characterized as partiallydeveloped supercritical inflow. In the turbulent shear region, the void fraction exhibits a Gaussian distribution about a maximum air concentration located slightly above the location of maximum bubble frequency (Resch et al., 1974; Chanson and Brattberg, 2000). The void fraction increases sharply towards unity as the free surface is approached. Accurate estimates of void fraction in the shear and interfacial regions are required to develop predictions of gas transfer rates, cavitation, and flow bulking (Wilhelms and Gulliver, 2005).

Several inherent difficulties inhibit accurate air bubble measurements in a multiphase flow, including signal interpretation uncertainty in turbulent flow regimes, bubble-probe interaction errors, and the detection of small bubbles (Boyer et al., 2002). Consequently, a relatively limited number of experiments in the literature characterize air bubble diameter throughout the hydraulic jump. Resch et al. (1974) analyzed hot-wire signals to estimate bubble diameter from air exposure time, and found the probability distribution function (PDF) of bubble diameter exhibits a log normal distribution with a peak around 5 $\mathrm{mm}$. A similar technique was used to show that maximum bubble diameter decreases with distance from the toe (Babb and Aus, 1981). Optical fiber probes have been used to characterize bubble diameter in the turbulent shear region as ranging from $2 \mathrm{~mm}$ to $12 \mathrm{~mm}$ for hydraulic jumps of $2<F r<8$ (Murzyn et al., 2005). Others have neglected to define a bubble diameter, and instead present bubble chord times, or the time spent by the bubble on a conductivity probe tip (Murzyn and Chanson, 2007; Chanson, 2007b). From these experiments, the bubble chord time PDF also exhibits a log normal distribution for a wide range of $F r$ jumps. As $F r$ is increased, the PDF becomes more skewed as both the maximum chord time and the number of small bubbles increase. Because of the simplifying assumptions employed in each of these techniques, measurements of bubble size in a hydraulic jump are typically regarded as approximate values (Babb and Aus, 1981).

The relationship between turbulence and bubble dynamics is not completely understood, as the intrusive nature of multiphase experimental studies limits the acquisition of instantaneous flow data. 
Formation of the shear layer begins at the toe where the impingement of the roller upon the upstream jet is the primary source of both entrained air and vorticity (Hornung et al., 1995; Dabiri and Gharib, 1997). Shear layer instabilities induced by toe oscillations produce spanwise vortices that travel downstream where, through pairing, they can increase in size from $2 d$ to $7 d$ (Long et al., 1991; Zhang et al., 2012). The strength of turbulence in the shear region is at a maximum near the toe (Resch and Leutheusser, 1972; Liu et al., 2004), leading to bubble breakup and corresponding with the location of highest bubble frequency (Murzyn et al., 2005). Recent studies have revealed positive correlations between air entrainment and turbulent fluctuations of the roller surface (Murzyn and Chanson, 2009; Chachereau and Chanson, 2011).

Hoyt and Sellin (1989) observed a braid-roll structure within the shear layer that contributes to air entrainment in the roller, and proposed the hydraulic jump be interpreted as a mixing layer with both transverse and streamwise coherence. Within a typical mixing layer, two parallel streams of fluid with differing velocities merge, resulting in coherent vortices whose evolution is governed by the natural or forced instabilities upstream of their conjunction. In the hydraulic jump, a recirculating roller region with negative velocity is situated above an impinging flow with positive velocity, and large local instabilities induced through the self-forcing action of recirculation and toe oscillations result in coherent vortices. Evidence of coherent vortical structures in mixing layers of free-shear flows is well documented (see e.g. Brown and Roshko, 1974; Hussain, 1986), but very few experimental observations exist for the classic hydraulic jump problem. The bulk of literature on coherent vortical structures in the hydraulic jump is based on qualitative visualization of primary spanwise vortices in high $(F r>2)$ Froude number jumps (Mossa and Tolve, 1998; Zhang et al., 2012), or, at most, experiments at low $(F r<2)$ Froude number with the addition of point velocity measurements (Misra et al., 2008; Rodríguez-Rodríguez et al., 2011).

Advances in computer power have made the numerical study of hydraulic jumps a viable companion to laboratory investigations. The first simulations captured free surface fluctuations in the liquid phase but ignored the contributions of air entrainment outright (Liu and Drewes, 1994; Chippada et al., 1994). Later, multiphase simulations implemented a phenomenological subgrid model that determined air entrainment based on turbulent fluctuations at the free surface (Souders and Hirt, 2004; Gonzalez and Bombardelli, 2005). These models, in line with numerous others (e.g. Carvalho et al., 2008; Abbaspour et al., 2009), have shown that Reynolds-Averaged Navier-Stokes (RANS) simulations with two-equation turbulence models, in combination with a Volume of Fluid (VOF) method for resolving the free surface, can capture the formation of the jump. Quantitative numerical results of void fraction distributions in a hydraulic jump have recently been produced using a subgrid air entrainment model (Ma et al., 2011; Cheng and Chen, 2011). However, these studies did not resolve the physical processes of bubble entrainment and breakup that dictate the void fraction profile, i.e., air entrainment was simulated based on inputs from experimental data or empirical relationships. Attempts to reproduce air entrainment directly through numerical simulation have been thus far unsuccessful (Lubin et al., 2009). This limitation restricts use of numerical simulations to instances where experimental verification of bubble diameters is readily available, which is not often the case.

A recent review of the state of aerated flow numerical simulations revealed a significant deficiency in model validation and a lack of valuable insights into relevant flow dynamics (Chanson, 2013). The aim of this paper is to present fully validated simulations of hydraulic jumps that inherently predict air-water flow characteristics without the use of experimental calibration. We also present the flow physics in terms of vortical structures observed in 3D numerical simulations, and their contributions to air-bubble transport. This paper is organized as follows. In Section 2, the numerical technique is described, the time dependence of the hydraulic jump is explored to determine an appropriate sampling rate and time for the numerical simulations, and a grid analysis is conducted to establish an appropriate computational cell size for capturing simulated air entrainment. In Section 3, validations of time-averaged velocity profiles, void fraction profiles, and bubble diameter are presented. Section 4 explores the flow physics in more detail by showing new evidence of vortex merging, the existence of streamwise vortices, the relationship between toe fluctuations and vortex evolution, and the similarities and differences between the hydraulic jump and a classic mixing layer. Conclusions are offered in Section 5. 


\section{Problem setup and numerical methodology}

\subsection{Problem setup}

Our simulation conditions were designed to model the experiment of Murzyn et al. (2005). A representation of their experimental setup is shown in Fig. 1. A recirculating laboratory flume $12 \mathrm{~m}$ long, $0.3 \mathrm{~m}$ wide, and $0.4 \mathrm{~m}$ high was used to create a quasi stable hydraulic jump. A sluice gate controlled the depth of the impinging jet, $d$, while a weir near the downstream outlet stabilized the jump and controlled the tailwater depth, $d_{t w}$. An RBI dual-tip probe optical phase-detection instrument was used to measure void fractions at various depths and distances downstream from the foot of the jump. The inflow exhibited a boundary layer thickness ranging from $0.2 d-0.4 d$, characterizing the jump as partially developed. No free surface aeration occurred upstream of the impingement point, and the incident flow was free of bubbles. The velocity of the upstream jet, $U_{o}$, and $d$ were adjusted to result in a variation of low Froude number jumps. The measurements at $F r=2.43,3.65$, and 4.82 are used for comparison in the present study.

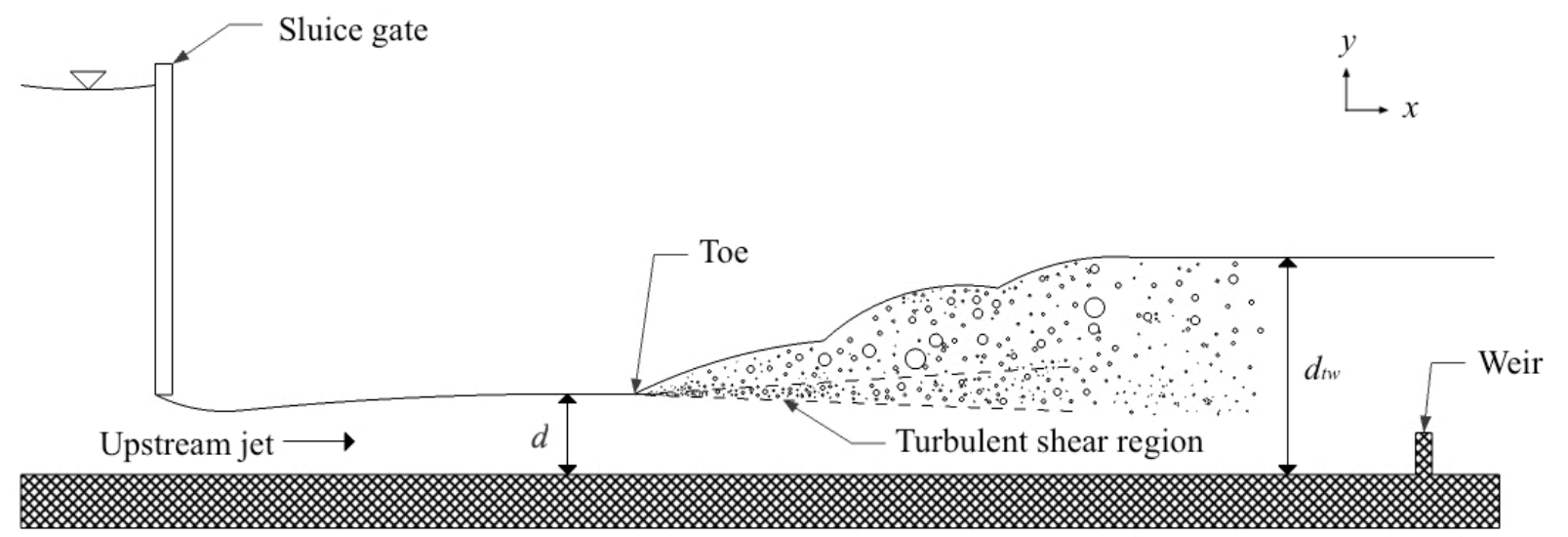

Fig. 1. Representation of the experimental set-up of Murzyn et al. (2005). Not to scale.

\subsection{Numerical methodology}

Numerical simulations are carried out using OpenFOAM ${ }^{\circledR}$, an open source computational fluid dynamics software package. Two and three-dimensional (2D and 3D) simulations are conducted assuming an unsteady, turbulent, incompressible flow. The evolution of the free surface is modeled using interFoam, a VOF solver designed for two immiscible, isothermal, interpenetrating fluids (OpenFOAM, 2012). The hydraulic jump is a complex problem to simulate due to the highly transient behavior of the toe and the turbulent entrainment and subsequent shearing of large air pockets in the roller. The interFoam solver has been shown to accurately resolve the physics of air entrainment and hydrodynamic breakup processes in a variety of fluid phenomena (Deshpande et al., 2012). To our knowledge, the present study is the first application of the solver to investigate the air entrainment characteristics of the hydraulic jump.

In the VOF approach, a single set of momentum equations is shared by the two phases, which are mathematically treated as a single continuum medium. The volume of fluid in each computational cell is tracked by an indicator function, $\gamma$, and the free surface evolution is governed by a transport equation for $\gamma$ in cells that contain a phase interface. The governing equations take the form 


$$
\begin{aligned}
& \nabla \cdot \boldsymbol{u}=0, \\
& \partial(\rho \boldsymbol{u}) \partial t+\nabla \cdot \rho \boldsymbol{u} \boldsymbol{u}=-\nabla p+\rho \boldsymbol{g}+\nabla \cdot \boldsymbol{\tau}+\sigma \kappa \nabla \gamma, \\
& \partial \gamma \partial t+\nabla \cdot(\gamma \boldsymbol{u})+\nabla \cdot(\boldsymbol{u} \boldsymbol{c} \gamma(1-\gamma))=0,
\end{aligned}
$$

where $\boldsymbol{u}$ is the fluid velocity vector, $\rho$ is the fluid density, $p$ is the pressure, $\boldsymbol{\tau}$ is the viscous stress tensor given as $\tau=2 \mu \boldsymbol{S}=2 \mu\left(0.5\left[(\nabla \boldsymbol{u})+(\nabla \boldsymbol{u})^{\mathrm{T}}\right]\right)$ where $\mu$ is the dynamic viscosity, $\sigma$ is the surface tension, $\kappa$ is the mean curvature of the free surface, $\gamma$ indicates the volume fraction of fluid in each computational cell, and $\boldsymbol{u}_{c}$ is an artificial "compressive velocity", which acts normal to the phase interface to counter numerical diffusion (Rusche, 2002; OpenFOAM, 2012).

The fluid domain consists of a mixture of phases, where each computational cell contains a phase fraction between $\gamma=1$, indicating the continuous fluid (liquid, $\varphi=0$ ), and $\gamma=0$, indicating the dispersed fluid (gas, $\varphi=1$ ). The fluid properties $\rho$ and $\mu$ are calculated based on the weighted contributions of the volume fraction of respective fluid as (Rusche, 2002)

$$
\begin{aligned}
& \rho=\rho l \gamma+\rho(1-\gamma), \\
& \mu=\mu l \gamma+\mu(1-\gamma) .
\end{aligned}
$$

Surface tension forces at the interface are represented using the continuum-surface-force model developed by Brackbill et al. (1992). A constant surface tension coefficient of $\sigma=0.07 \mathrm{~N} / \mathrm{m}$ was maintained for all simulations, and curvature is computed based on the volume fraction gradient. The model has shown good performance in free-surface flows (Krishna and van Baten, 1999; Deshpande et al., 2012).

Numerical simulations of the hydraulic jump utilizing a two equation $k-\varepsilon$ formulation have shown good agreement with laboratory measurements (Liu and Drewes, 1994; Zhao et al. 2004; Carvalho et al., 2008). To apply this approach, Eqs. 1 and 2 are Reynolds averaged, resulting in a Reynolds stress that represents the transport and dissipation of energy due to turbulence. Next, $\mu$ is replaced by an effective viscosity, $\mu_{\text {eff, }}$ where

$$
\mu e f f=\mu+\mu t
$$

and $\mu_{t}$ is the turbulent eddy viscosity. Eddy viscosity is then related to turbulent kinetic energy, $k$, and energy dissipation, $\varepsilon$, through a constitutive relationship

$$
\mu t=\rho C \mu k 2 \epsilon,
$$

where $C_{\mu}$ is a variable model parameter sensitized to the flow using the following relations (Shih et al., 1995):

$$
\begin{aligned}
& C \mu=14+A s \quad * k \epsilon, \quad A s=6 \cos \phi, \quad \phi=13 \arccos 6 W, \quad W=\text { SijSjkSkiS3 }, \\
& S=\operatorname{SijSij~}, \quad U^{*}=S i j S i j+\Omega i j \Omega i j, \quad \Omega i j=12 \partial u i \partial x j-\partial u j \partial x i .
\end{aligned}
$$

The transport of $k$ and $\varepsilon$ are computed using

$$
\begin{aligned}
& \partial(\rho k) \partial t+\partial(\rho k \boldsymbol{u}) \partial \boldsymbol{x}=\partial \partial \boldsymbol{x} \mu+\mu t \sigma k \partial k \partial \boldsymbol{x}+G k-\rho \epsilon \\
& \partial(\rho \epsilon) \partial t+\partial(\rho \epsilon \boldsymbol{u}) \partial \boldsymbol{x}=\partial \partial \boldsymbol{x} \mu+\mu t \sigma \epsilon \partial \epsilon \partial \boldsymbol{x}+\rho C 1 \boldsymbol{S} \epsilon-\rho C 2 \epsilon 2 k+v \epsilon
\end{aligned}
$$


where $\sigma_{k}$ and $\sigma_{\varepsilon}$ are model constants equal to 1 and 1.2, respectively, $G_{k}$ represents turbulent kinetic energy generation from the mean velocity gradients,

$$
G k=2 \mu t S i j S i j,
$$

$C_{1}$ is a variable model parameter,

$$
C 1=\max 0.43, \eta \eta+5, \eta=k \epsilon 2 S i j S i j,
$$

$C_{2}$ is a model constant equal to 1.9 , and $v$ is the kinematic viscosity (OpenFOAM, 2012). In contrast to the standard $k-\varepsilon$ closure scheme, the realizable $k-\varepsilon$ scheme ensures the positivity of normal stresses by sensitizing $C_{\mu}$ to the mean strain rate, mean rotation rates, and the turbulence fields in a matter consistent with the physics of turbulent flows. The realizable scheme exhibits improved performance over the standard $k-\varepsilon$ scheme in shear flows (Zhu and Shih, 1994; Kim et al., 1999), recirculating flows (Qian et al., 2009), and when air bubble entrainment is a relevant flow characteristic (Zidouni et al. 2010).

To discretize the transient terms, a Crank-Nicolson scheme was used with an off-centering coefficient of 0.5 for all but the smallest grid size, where a coefficient of 0.9 was employed to improve the numerical stability of the simulations as cell size was reduced. Spatial discretization was carried out using a second order, Gauss linear discretization scheme. Cells containing a volume fraction interface are discretized with a second order blended difference scheme and a van Leer limiter (Rusche, 2002; OpenFOAM, 2012). The Pressure Implicit Splitting of Operators (PISO) algorithm was used for coupling the pressure and velocity, while the time step was limited by a maximum Courant number of 0.5 . The discretization scheme maintained a second order global accuracy.

\subsection{Simulation conditions}

As an example, Fig. 2 shows the computational domain and grid for $F r=4.82$. Boundary conditions are given in Eqs. 12-17. On the left hand boundary $\left(\Omega_{i l}\right)$, an inlet flow of depth $d$ was set to match experimental conditions for each $F r$ jump $(d=0.046 \mathrm{~m}, 0.032 \mathrm{~m}$, and $0.021 \mathrm{~m}$ for $F r=2.43,3.65$, and 4.82 , respectively), a wall was placed above the inlet up to $0.2 \mathrm{~m}$ to represent the sluice gate $\left(\Omega_{w l}\right)$, and the remaining depth was open to the atmosphere $\left(\Omega_{o l}\right)$. The top $\left(\Omega_{o t}\right)$ and right $\left(\Omega_{o r}\right)$ boundaries were also open to the atmosphere. The bottom boundary was a no-slip wall $\left(\Omega_{w b}\right)$, and an overshot weir was placed near the downstream end of the mesh to set the tailwater. The entire domain was bounded by a depth of $0.4 \mathrm{~m}$, and a length of $2.6 \mathrm{~m}$ for the $F r=2.43$ simulation, $2.9 \mathrm{~m}$ for the $F r=3.65$ simulation, and $2.1 \mathrm{~m}$ for the $F r=4.82$ simulation. The initial grid was a structured, uniform mesh. Successive, local grid refinements were made from the left hand boundary to a minimum of $10 \mathrm{~d}$ past the final measurement location. The goal of the present study is to capture the air entrainment characteristics at the toe and subsequent bubble transport downstream. The numerical domain was optimized to force a jump to occur in the first $0.5 \mathrm{~m}$ of the domain, ensuring all air entrainment, bubble breakup, and bubble transport corresponding with the experimental measurement locations remain fully contained in the finest mesh. Within this region, the first grid nodes off the bottom wall were within $60<y^{+}<80$ for all four simulation cases. These locations are within the log layer (Pope, 2002), and consistent with the guidance range of 50 $<y^{+}<100$ obtained through validation studies using wall models developed and employed in OpenFOAM (Jasak, 1996). A wall function was used to compute eddy viscosity near the wall to ensure the turbulent quantities were properly represented, and the boundary layer was accurately modeled. 


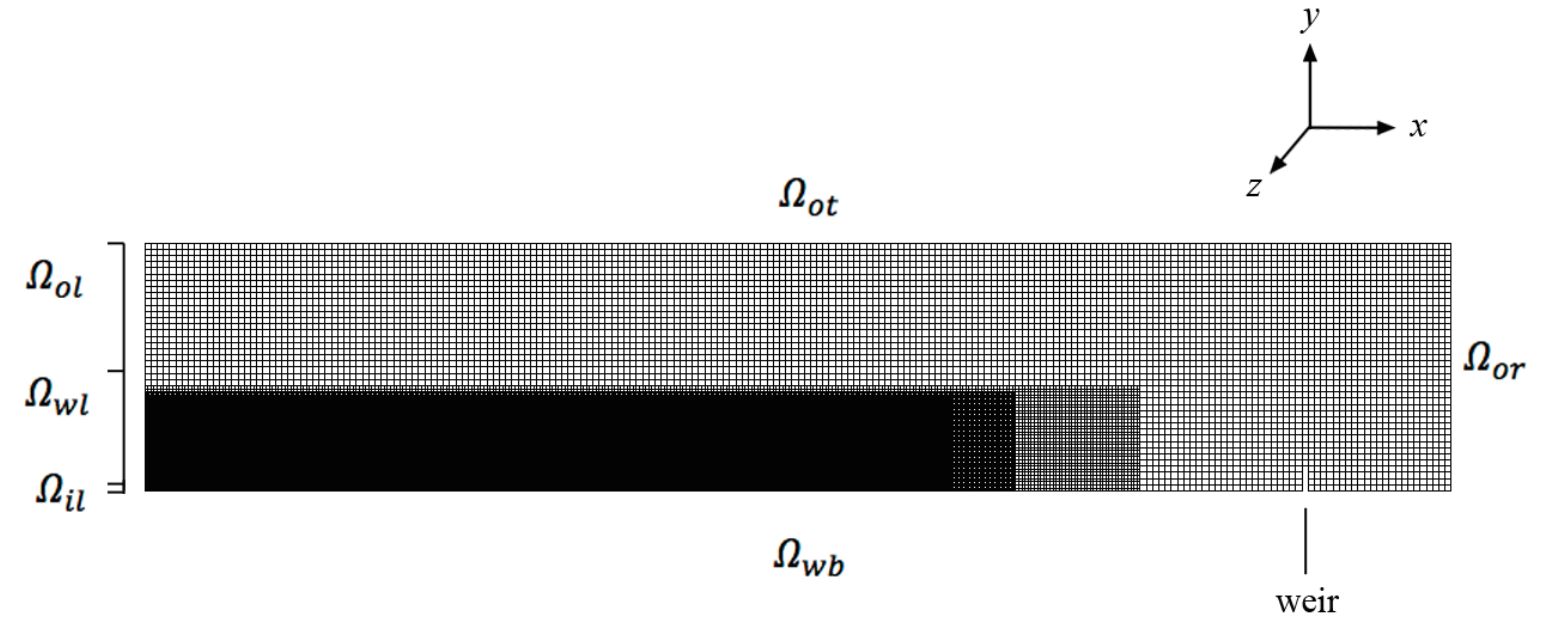

Fig. 2. 2D computational grid for the case of $F r=4.82$.

$\begin{array}{llll}\Omega i l: & \boldsymbol{n} \cdot \nabla p=-\boldsymbol{n} \cdot \nabla \rho \boldsymbol{g} \cdot \boldsymbol{x} ; & \boldsymbol{u}=U o, 0 ; & \gamma=1 ; \\ \Omega w l: & \boldsymbol{n} \cdot \nabla p=-\boldsymbol{n} \cdot \nabla \rho \boldsymbol{g} \cdot \boldsymbol{x} ; & \boldsymbol{u}=0,0 ; & \boldsymbol{n} \cdot \nabla \gamma=0 ; \\ \Omega o l: & \boldsymbol{n} \cdot \nabla p=0 ; & \boldsymbol{n} \cdot \nabla \boldsymbol{u}=0 ; & \boldsymbol{n} \cdot \nabla \gamma=0 ; \\ \Omega o t: & p+\quad 2=0 ; & \boldsymbol{n} \cdot \nabla \boldsymbol{u}=0 ; & \boldsymbol{n} \cdot \nabla \gamma=0 ; \\ \Omega o r: & \boldsymbol{n} \cdot \nabla p=0 ; & \boldsymbol{n} \cdot \nabla \boldsymbol{u}=0 ; & \boldsymbol{n} \cdot \nabla \gamma=0 ; \\ \Omega w b: & \boldsymbol{n} \cdot \nabla_{p}=-\boldsymbol{n} \cdot \nabla \rho \boldsymbol{g} \cdot \boldsymbol{x} ; & \boldsymbol{u}=0,0 ; & \boldsymbol{n} \cdot \nabla \gamma=0 .\end{array}$

A constant horizontal velocity, $U_{o}$, was prescribed at the flow inlet to match experimental conditions. Outlets were prescribed a zero gradient velocity condition, $\boldsymbol{n} \cdot \nabla \boldsymbol{u}=0$, where $\boldsymbol{n}$ is the normal surface vector, and all walls were modeled as no-slip. Velocity in the first near-wall cell was calculated from the momentum equation, with the turbulent viscosity acting as a source term to compensate for the unresolved shear stress at the wall (Rusche, 2002). The upstream jet traveled approximately 15-20d downstream from the inlet before encountering the tailwater, which was a sufficient length to ensure the velocity profile developed a boundary layer thickness of between $0.2 d$ and $0.4 d$, and could be characterized as partially developed. This thickness was consistent with the experimental boundary layer thickness estimated at the vena contracta, i.e. the location where the streamlines are no longer contracting. Note that experimental data exactly at the inlet is unavailable, and thus we made no attempt to model the specific velocity exactly at the inlet. No contraction was allowed at the simulated inlet, ensuring the orientation of the simulated upstream flow streamlines was identical to those at the experimental vena contracta, where data analysis was typically performed in experiments.

Total pressure at the top outlet, $p+\rho \boldsymbol{u}^{2}$, was set equal to 0 and $p$ was calculated from $\boldsymbol{u}$ on the boundary. Walls and outlets were prescribed with a buoyant pressure condition. Turbulence parameters $k$ and $\varepsilon$ were modeled using a zero gradient condition at all outlets while solid wall boundaries were modeled using a logarithmic wall function. Values of $k$ and $\varepsilon$ at the inlet were assigned assuming 5\% turbulence intensity and a turbulent length scale of $10 \%$ of the inlet depth. These values, together with the 15-20d downstream distance between the inlet and toe, ensure the boundary layer of the upstream jet was partially developed to match the experiments by Murzyn et al. (2005). 
A 3D simulation was also conducted for $F r=4.82$. The front and back planes, $\Omega_{f p}$ and $\Omega_{b p}$, respectively, were modeled as a free-slip surface. This treatment is identical to Eq. (14) for scalars and tangential components of velocity vectors, while the normal components of velocity vectors are set equal to 0 . The grid was similar to Fig. 2 with a width of $0.1 \mathrm{~m}$ extended in the $z$ direction. This width was chosen to model the interior of the experimental domain, where side wall effects are negligible (Chanson, 2007a). The mean motion of bubbles is highly influenced by spanwise vortices, which are constrained by the free surface, and to a small degree, streamwise vortices. Domain boundaries were chosen to ensure spanwise vortices are fully resolved in both $2 \mathrm{D}$ and $3 \mathrm{D}$ simulations, and streamwise vortex pairs are fully resolved in the $3 \mathrm{D}$ simulation. For perspective, the maximum mean velocity in the lateral direction $(z)$ on an $x-y$ plane through the center of the domain is less than $1 \%$ of the inlet velocity. As a comparison, the maximum vertical $(y)$ and streamwise $(x)$ velocities are nearly $20 \%$ and $100 \%$, respectively, of the inlet velocity. The maximum bubble diameter is approximately $10 \%$ to $20 \%$ of the domain width, and the Sauter mean diameter is approximately $5 \%$ of the domain width. Given this ratio, and considering the lateral motion of fluid is minimal compared with streamwise and vertical motion, the free motion of bubbles is not constrained in the lateral direction during their residence time in the jump.

\subsection{Time dependence}

The void fraction measurements of Murzyn et al. (2005) were obtained using optical fiber probes with a sampling rate of $1 \mathrm{MHz}$ and a sampling time of $120 \mathrm{~s}$. Though a range of sampling rates and times have been reported in the literature, Chanson and Brattberg (2000) found no difference in air concentration distributions at sampling times longer than $10 \mathrm{~s}$. On the other hand, computational restraints allowed for a maximum sampling rate of $20 \mathrm{~Hz}$, thus the sampling time dependence of the simulations was analyzed to find a balance between computational cost and modeling accuracy. The integration of time-averaged void fraction over depth, i.e. $\Sigma{ }_{j} \Delta y_{j i}$, was chosen as the principal indicator of convergence, where $y$ indicates the vertical position, $i$ represents the horizontal index, $j$ represents the vertical index, and is the time-averaged void fraction. A vertical integration is representative of the average amount of air entrained into the flow over time, and therefore serves as a gauge of accuracy for air entrainment.

A visual observation shows the average void fraction profile approaching a constant shape after just 1 s of sampling time at $20 \mathrm{~Hz}$ (Fig. 3). To quantify average air entrainment from our simulations, vertical data points were obtained at four downstream horizontal locations matching those of Murzyn et al. (2005). A void fraction of 0.5 was assumed to be the location of the free surface and served as a cutoff for depth integration. The value of $\Sigma{ }_{j} \Delta y_{j i}$ for each 2D simulation was obtained every $0.05 \mathrm{~s}$, and a time average was carried out over $20 \mathrm{~s}$. The relative error in $\Sigma{ }_{j} \Delta y_{j i}$ after $5 \mathrm{~s}, 10 \mathrm{~s}$, and $15 \mathrm{~s}$ of time averaging was computed using $\Sigma{ }_{j} \Delta y_{j i}$ obtained after a $20 \mathrm{~s}$ sampling time. For the $F r=4.82$ case, the relative error after $5 \mathrm{~s}, 10 \mathrm{~s}$, and $15 \mathrm{~s}$ sampling times was $0.076,0.054$, and 0.007 , respectively. The relative error in average entrained air after $15 \mathrm{~s}$ of sampling time showed a similar trend for all $F r$ jumps, thus a sampling time of $15 \mathrm{~s}$ is used in the following analyses. A $15 \mathrm{~s}$ sampling time represents $7.5-30$ toe pulsations for hydraulic jumps in the range of Froude number and Reynolds number considered in this study (Chanson and Gualtieri, 2008). 


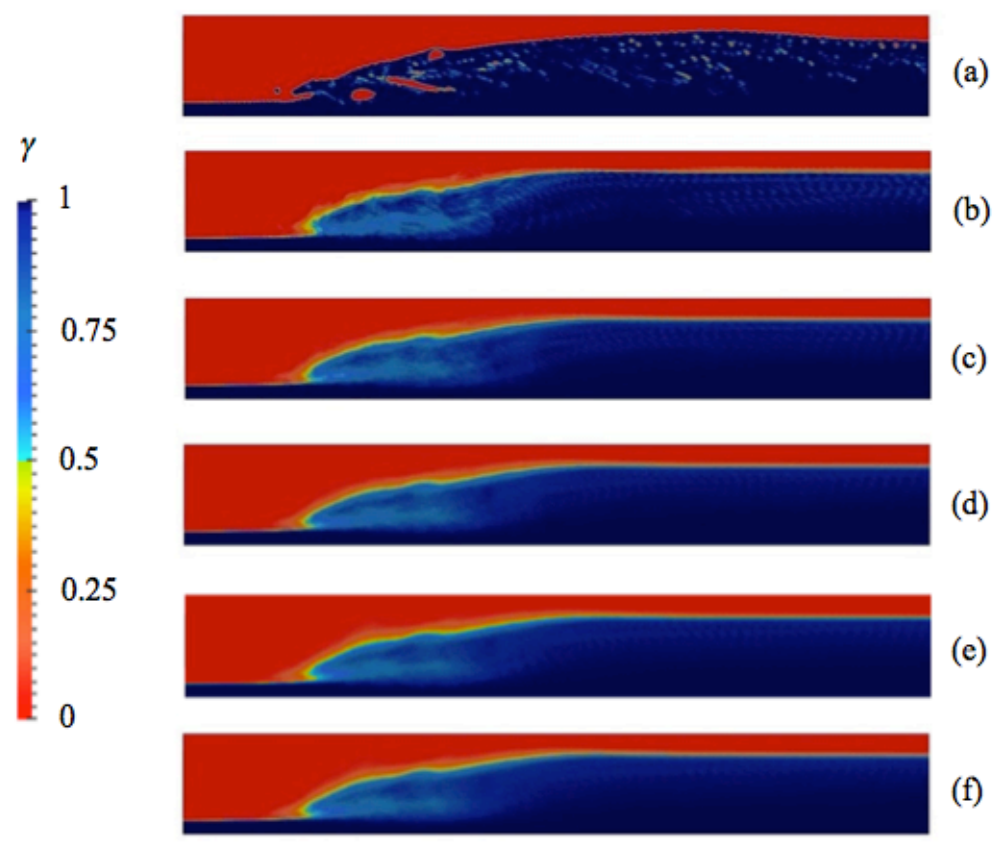

Fig. 3. Time-averaged volume fraction field for a $2 \mathrm{D}$ simulation of $F r=4.82$ after $0 \mathrm{~s}$ (i.e., instantaneous field in this case) (a), $1 \mathrm{~s}(\mathrm{~b}), 5 \mathrm{~s}(\mathrm{c}), 10 \mathrm{~s}(\mathrm{~d}), 15 \mathrm{~s}(\mathrm{e})$, and $20 \mathrm{~s}$ (f).

\subsection{Grid analysis}

Void fraction dependence on cell density was measured at each grid refinement level. The coarsest grid was a structured, uniform mesh with cells of length $10 \mathrm{~mm}$, roughly $0.3 d-0.5 d$. At subsequent refinement levels the cell length, $\Delta x$, was reduced by half in the region of interest (Fig. 2). Again, $\Sigma{ }_{j} \Delta y_{j i}$ was chosen as the principal indicator of convergence, and relative error was computed with respect to the total entrained air obtained by Murzyn et al. (2005). The results are listed in Table 1. A relative error of less than $10 \%$ of experimental values was deemed acceptable for purposes of evaluation. This was obtained at $\Delta x=1.25 \mathrm{~mm}$ for $2 \mathrm{D}$ simulations and at $\Delta x=2.5 \mathrm{~mm}$ for the 3D simulation. Therefore, we have chosen to use $\Delta x=1.25 \mathrm{~mm}$ for all cases. At this grid size, relative error in the 3D simulation was $35 \%$ of the relative error from the $2 \mathrm{D}$ simulation, while the computational time was increased by a factor of 341. If $\Delta x$ is further reduced from $1.25 \mathrm{~mm}$ to $0.625 \mathrm{~mm}$, the relative error in the $F r=4.822 \mathrm{D}$ case is reduced from $3.7 \%$ to $1.0 \%$, but the computational time is increased substantially by a factor of 29 .

A significant improvement is seen in 2D simulations when the grid size is reduced from $\Delta x=2.5 \mathrm{~mm}$ to $\Delta x=1.25 \mathrm{~mm}$ (Grid 4). At this grid size the largest bubbles in the flow are represented by at least eight computational cells, producing a bubble diameter to grid ratio that is sufficient to capture surface deformations caused by the interaction of vortices and the bubble interface (Tomiyama et al., 1993). These interactions occur frequently in the turbulent shear layer, leading to significant breakup, i.e., the creation of two or more daughter bubbles from one parent bubble. Within the shear layer, the largest bubbles and entrained air pockets are found near the toe, and vary from $10-20 \mathrm{~mm}$ in diameter (Murzyn et al., 2005). A bubble diameter to grid size ratio of eight is achieved for $10 \mathrm{~mm}$ bubbles with Grid 4 . When this occurs the largest bubbles are sheared more effectively by the underlying turbulence, their internal flow fields are resolved, and their mean motion within the shear layer is better represented. Resolution of the smaller satellite bubbles produced during breakup is improved, resulting in a greater bubble population and a wider range of bubble diameters than what is observed on coarser grids. The 
subsequent transport and turbulent dispersion of bubbles produced via breakup also improves the simulation result of average entrained air downstream.

\begin{tabular}{|c|c|c|c|c|c|c|c|c|c|c|c|c|c|}
\hline \multirow[t]{2}{*}{ Grid } & \multirow{2}{*}{$\begin{array}{c}\operatorname{Max} x \\
\text { (m) }\end{array}$} & \multicolumn{4}{|c|}{ Grid size } & \multicolumn{4}{|c|}{ CPU time (h) } & \multicolumn{4}{|c|}{ Relative error } \\
\hline & & $F r=2.43$ & 3.65 & $\begin{array}{c}4.82 \\
2 \mathrm{D} \\
\end{array}$ & $\begin{array}{c}4.82 \\
3 \mathrm{D} \\
\end{array}$ & 2.43 & 3.65 & $\begin{array}{c}4.82 \\
2 \mathrm{D} \\
\end{array}$ & $\begin{array}{c}4.82 \\
3 \mathrm{D} \\
\end{array}$ & 2.43 & 3.65 & $\begin{array}{c}4.82 \\
2 \mathrm{D} \\
\end{array}$ & $\begin{array}{c}4.82 \\
3 \mathrm{D} \\
\end{array}$ \\
\hline 1 & 0.01 & $2.6 \mathrm{E}+04$ & $2.3 \mathrm{E}+4$ & $1.7 \mathrm{E}+4$ & $8.4 \mathrm{E}+4$ & 0.3 & 0.6 & 0.5 & 1.9 & 0.622 & 0.422 & 0.689 & 0.391 \\
\hline 2 & 0.005 & $5.7 E+04$ & $6.0 \mathrm{E}+4$ & $4.3 \mathrm{E}+4$ & $2.7 \mathrm{E}+5$ & 2.1 & 1.8 & 1.2 & 33 & 0.428 & 0.394 & 0.511 & 0.240 \\
\hline 3 & 0.0025 & $1.4 \mathrm{E}+05$ & $1.6 \mathrm{E}+5$ & $1.2 \mathrm{E}+5$ & $1.5 \mathrm{E}+6$ & 13 & 13 & 11 & 1,170 & 0.344 & 0.359 & 0.396 & 0.081 \\
\hline 4 & 0.00125 & $4.2 \mathrm{E}+05$ & $4.7 \mathrm{E}+5$ & $3.7 \mathrm{E}+5$ & $1.0 \mathrm{E}+7$ & 349 & 388 & 289 & 98,496 & 0.013 & 0.064 & 0.037 & 0.013 \\
\hline 5 & 0.000625 & & & $1.1 \mathrm{E}+6$ & & & & 8,361 & & & & 0.010 & \\
\hline
\end{tabular}

Table 1. Relative error in average void fractions, calculated with respect to the time-averaged void fractions of Murzyn et al. (2005). CPU time is obtained by multiplying the total simulation time by the number of processors. 


\section{Air and water flow characteristics}

In this section, the 2D and 3D numerical results are presented and compared with the experimental results of Murzyn et al. (2005). Ideally, validation of simulated aerated flows should include comparisons of velocity, void fraction, turbulence intensity, and bubble characteristics with experimental measurements (Chanson, 2013). For all simulations, average void fraction and bubble size are obtained from the volume fraction field. To the authors' knowledge, the validation of bubble diameter simulated without the use of an ad hoc subgrid bubble model is the first reported in the literature for a hydraulic jump. Based on the available experimental data, a direct comparison of turbulence intensity and mean velocity is not possible. Nevertheless, we compare some mean velocity characteristics with those obtained experimentally from similar Fr jumps.

\subsection{Velocity}

The mean horizontal velocity flow structure for $F r=4.82$ 3D is shown in Fig. 4. For the 3D validation, all values are obtained along an $x-y$ plane located on the channel centerline $(z=2.5 d)$. The downstream measurement location is given by $x$ - $x_{\text {toe }}$, where $x_{\text {toe }}$ is the average location of the toe, and $x$ is the downstream location. Velocity profiles compare favorably to the velocity field of similar $\mathrm{Fr}$ jumps obtained experimentally (Fig. 2 of Liu et al., 2004; Fig. 7 of Lin et al., 2012). Maximum velocity is observed at the toe and remains slightly above the wall moving downstream. The shear region begins immediately above the location of maximum velocity, denoted by the dashed black line, and extends vertically to the dashed white line, which denotes the minimum positive horizontal velocity $(u=0$ in the roller).

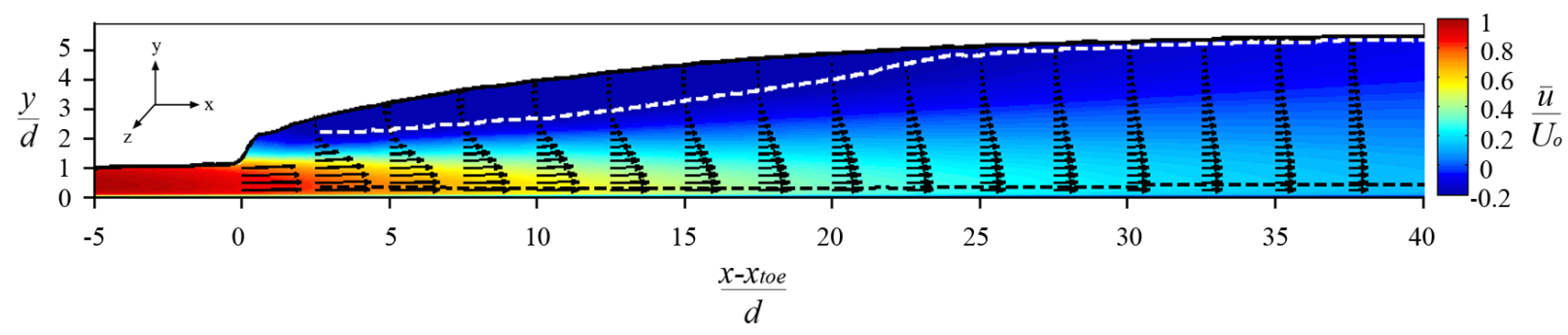

Fig. 4. Color contours representing the average horizontal velocity for $F r=4.823 \mathrm{D}$ beneath the mean free surface $(\gamma=0.5)$ in the center vertical plane. White and black dashed lines represent the location of minimum and maximum mean horizontal velocity, respectively, and arrows depict $(u, v)$ velocity vectors.

Maximum velocity decays exponentially with increasing streamwise distance from the toe (Fig. 5). Simulation data follow the exponential relationship

$$
\text { uumax }=\exp -\text { Ax-xtoed, }
$$

with $A=0.043$ for 2D data, and $A=0.030$ for 3D data, in agreement with the experimentally observed $A$ $=0.028$ for jumps with $F r>5$ (Chanson, 2010). The 2D numerical data exhibit a similar scatter about the exponential trend line as lower Fr jumps observed in experiments (Fig. 3.19 of Murzyn and Chanson, 2007; Fig. 11 of Chanson, 2010). The maximum velocity in 3D for $F r=4.82$ is approximately $10 \%$ higher than $2 \mathrm{D}$ at comparable locations within the shear layer. This is thought to be attributable to resolution of 3D vortex dynamics, as described in Section 4. 


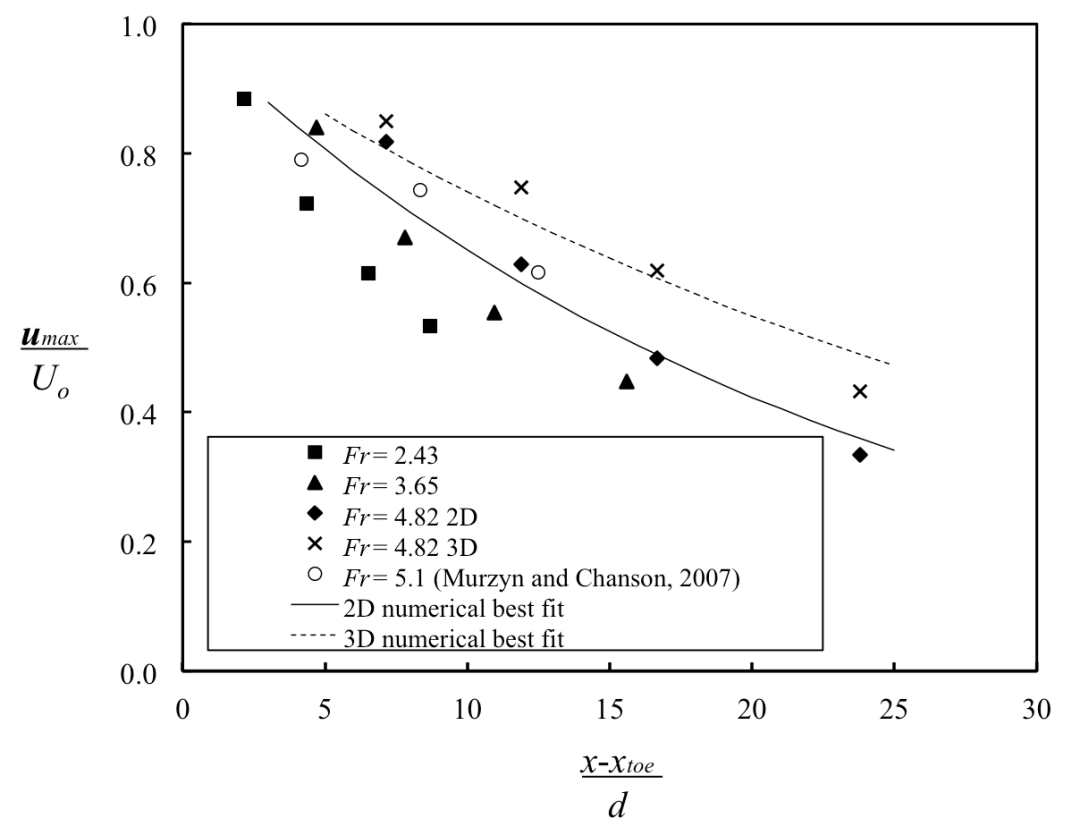

Fig. 5. Maximum velocity in the shear layer, normalized by upstream jet velocity, at multiple dimensionless downstream locations.

\subsection{Void fraction}

A quantitative representation of average void fraction is shown in Figs. 6-8. Each plot shows simulated and experimental time-averaged void fractions as a function of depth normalized by the upstream jet depth.
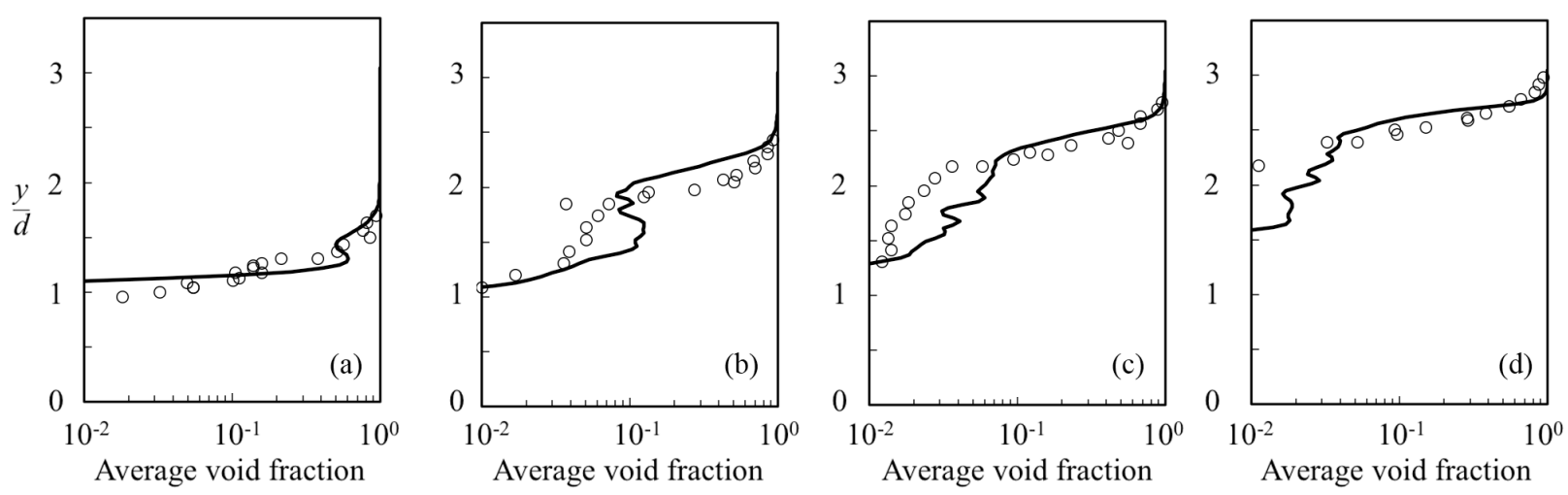

Fig. 6. Average void fraction profiles for $F r=2.43$ at $x$ - $x_{t o e}=2.17 d$ (a), $4.35 d(\mathrm{~b}), 6.52 d$ (c), and $8.7 d$ (d). 'O' denotes experimental average void fraction (Murzyn, 2013); ' — ' denotes simulated average void fraction. 

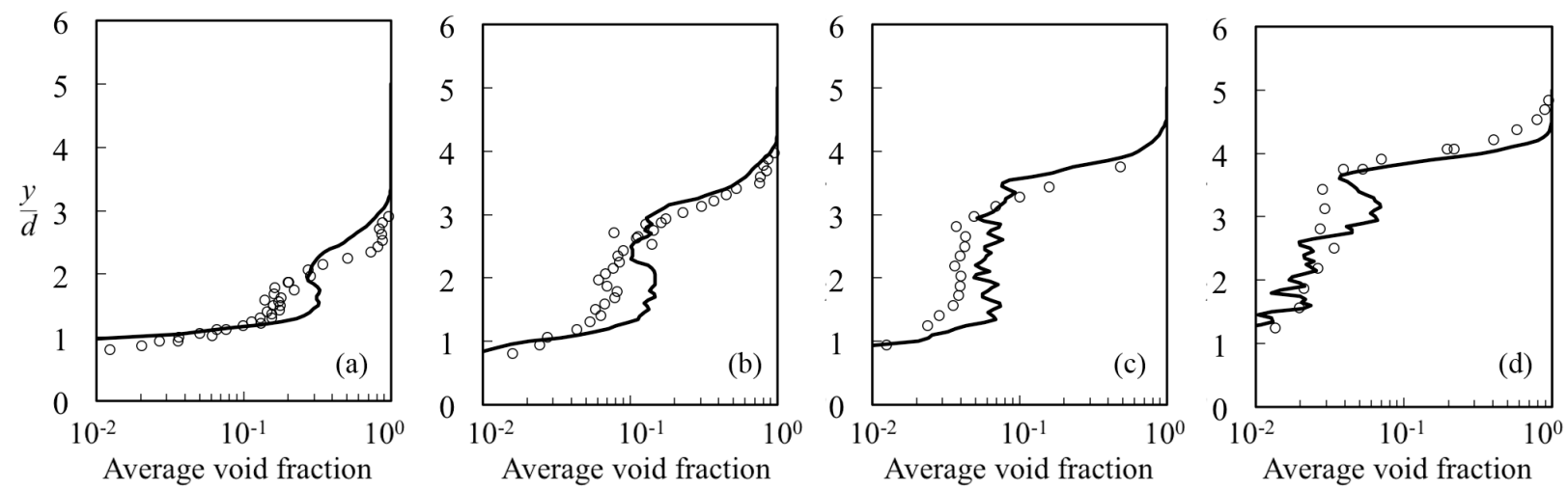

Fig. 7. Average void fraction profiles for $F r=3.65$ at $x-x_{\text {toe }}=4.69 d$ (a), $7.81 d$ (b), $10.94 d$ (c), and 15.6d (d). ' 0 ' denotes experimental average void fraction (Murzyn, 2013); ‘ ' denotes simulated average void fraction.
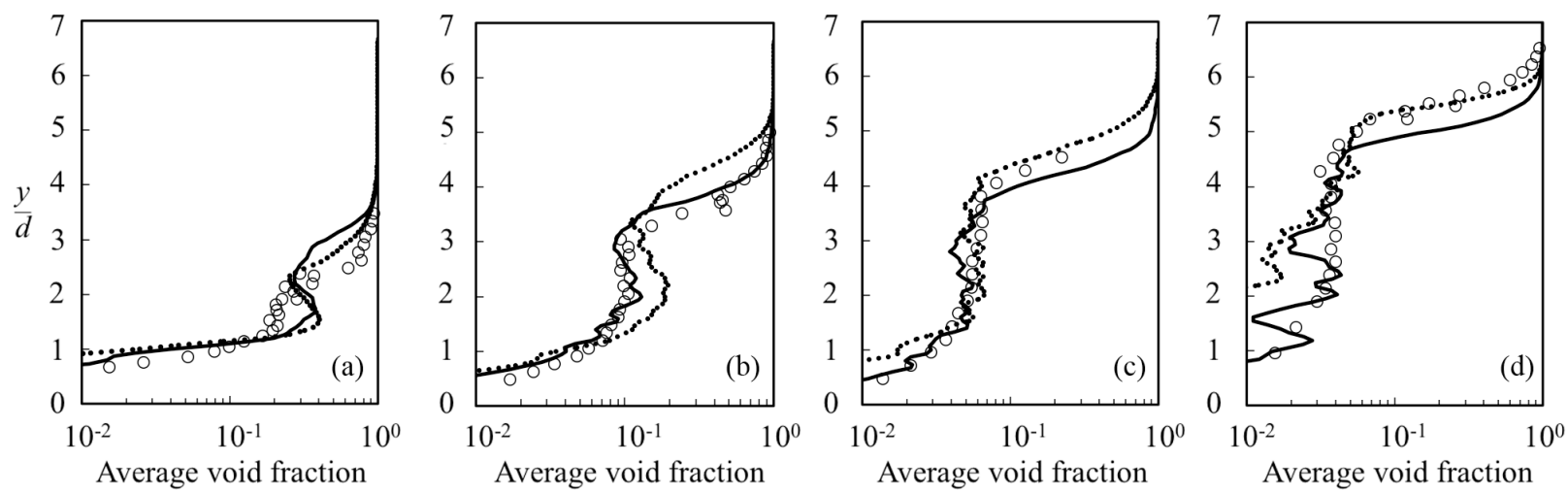

Fig. 8. Average void fraction profiles for $F r=4.82$ at $x-x_{\text {toe }}=7.14 d$ (a), $11.9 d$ (b), 16.67d (c), and 23.8d (d). 'O' denotes experimental average void fraction (Murzyn, 2013); '----' denotes 2D simulated average void fraction; ' ' denotes 3D simulated average void fraction.

Ascending at each location a rise in void fraction leads to a maximum air concentration within the shear region, above which void fraction begins to decrease. The shape is similar to a Gaussian solution to the advection-diffusion equation for the void fraction as described by Chanson (1996). The shape is more pronounced for higher $\mathrm{Fr}$ jumps, where air entrainment at the toe is increased. The simulation tends to predict higher void fractions in the shear region near the toe of the jump (Figs. 7-9, a and b). The turbulent action of the shear layer leads to instantaneous bubble breakup and coalescence, characterized by a continuous deformation of a moving interface. Regions exhibiting this flow structure are susceptible to higher uncertainties in both experiments and simulations. Despite the numerical challenges, average simulated air concentration rarely deviates greater than $10 \%$ from experiments. Moving downstream, void fractions show good agreement with experiments in the turbulent shear region (Figs. 7-9, c and d), indicating the simulations captured the entrainment of air at the toe, the breakup of air pockets in the shear layer, the rise of large bubbles out of the flow, and the transport of small bubbles downstream.

In the upper layer of the jump, breaking waves and turbulent ejections of fluid are the dominating mechanisms of air entrainment, and the void fraction profile increases sharply to $\varphi=1$. At nearly all simulation locations, this abrupt increase is captured at an elevation matching the experiments. Near the toe of the jump, the simulation tends to over predict the average free surface elevation by $0.2 d-0.5 d$ for higher $F r$ number jumps (Figs. 8-9, a). These increased elevations tend to match locations with higher 
void fraction in the shear layer, indicating that the presence of air in the shear layer has a bulking effect on the flow. Moving downstream, the simulation is accurate in capturing the upper void fraction profile, where free surface fluctuations are severely suppressed.

The longitudinal structure of average void fraction compares favorably with experimental values (Fig. 9 and Fig. 10). The elevation of maximum void fraction, $y_{C \max }$, versus horizontal location within the jump shows a linear relation with a gradient of 0.112 , in close agreement with 0.108 and 0.102 measured by Chanson and Brattberg (2000) and Murzyn et al. (2005), respectively. Maximum void fractions at each horizontal location decay with distance from the toe, in line with experiments. As $\mathrm{Fr}$ is increased and the jump transitions from oscillating to steady, simulated $C_{\max }$ and $y_{C \max }$ tend to improve towards the experimental values.

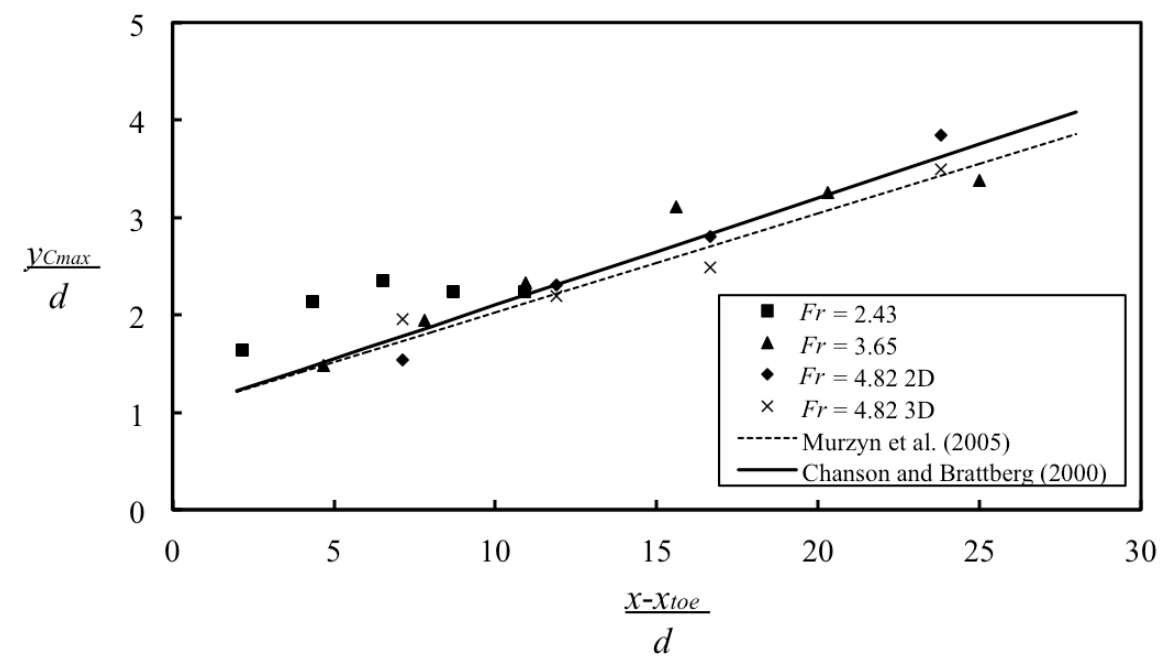

Fig. 9. Elevation of maximum air concentration, normalized by upstream jet depth, at multiple dimensionless downstream locations.

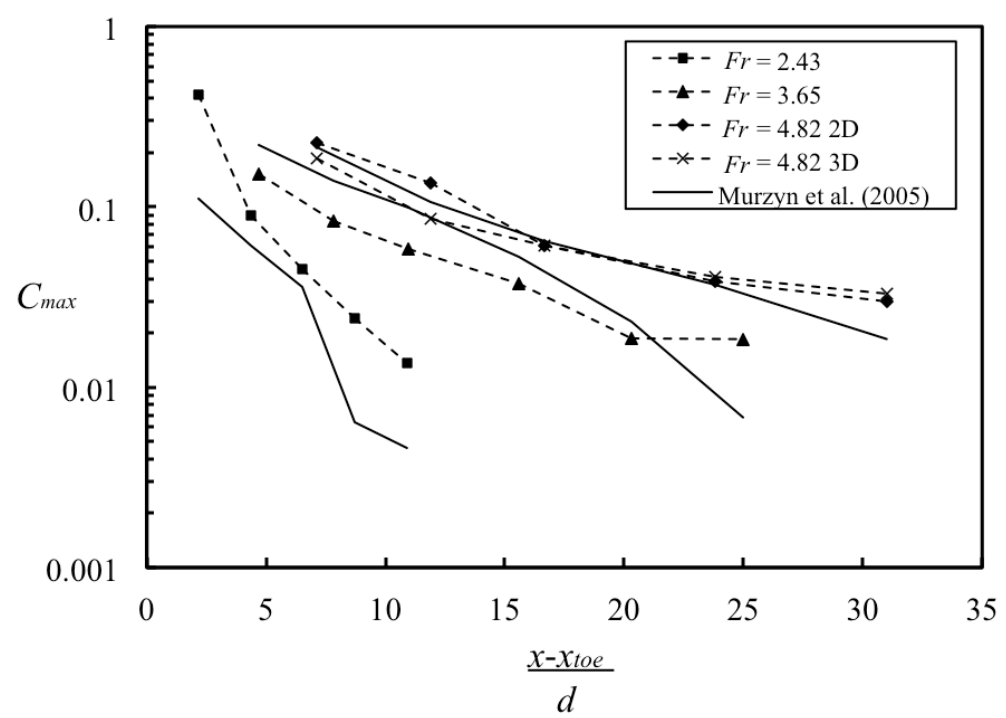

Fig. 10. Maximum air concentration at multiple dimensionless downstream locations.

Void fraction profiles and longitudinal structure for $F r=4.82$ in 2D and 3D show similarity throughout the jump. The largest improvement in 3D simulations is seen near the toe of the jump (Fig. 
8b). The 3D simulation improves void fraction predictions in the shear layer, and consequently the average free surface elevation shows improvement over the 2D simulation. The resolution of additional turbulent processes, vortex dynamics, and vortex interactions discussed in Section 4 is believed to be responsible for this improvement.

\subsection{Bubble diameter}

Validation of air entrainment is achieved through quantification of a Sauter mean diameter, i.e., the diameter of a single spherical bubble with the equivalent volume to surface area ratio of an ensemble of bubbles. A proper determination of Sauter mean diameter demands a sampling rate and sampling frequency at which an experimental apparatus can capture all relevant bubble characteristics. However, experimental sampling rates and sampling times required to achieve a stable bubble frequency count are significantly higher than what is computationally feasible (Chanson and Brattberg, 2000; Murzyn et al., 2005). A sensitivity analysis has shown that bubble count rates are underestimated for sampling rates below $5 \mathrm{kHz}$ and sampling times below 30 to 40 seconds (Chanson, 2007a). Due to the unrealistic computational demands of a $5 \mathrm{kHz}$ sampling rate, any numerical estimate of Sauter mean diameter will underrepresent bubble frequency. However, some insights into bubble size can be achieved through an analysis of the instantaneous void fraction field.

The assumption by experimentalists is that bubbles within the jump are spherical and detection instrumentation intercepts a bubble along its diameter (Resch et al., 1974; Babb and Aus, 1981; Waniewski et al., 2001). To simulate this technique, a contour map of individual bubbles is created for each time step through a second order interpolation of a 2D $\gamma$ field about a particular $\gamma$ value. In 3D, the $\gamma$ field on an $x-y$ plane through the centerline of the domain is analyzed, as in the experimental analysis of Murzyn et al. (2005). An analysis of various contour values shows a linear interpolation about $\gamma=0.95(\varphi$ $=0.05 \%$ ) produces the maximum bubble count, bubble frequency, and the proper distribution of bubble sizes while visually representing the bubble field of a hydraulic jump. Fig. 11 shows the $2 \mathrm{D}$ contour field of bubbles and the 3D flow field from side and cross-section views. Larger pockets of air are present at the toe, while smaller bubbles are visible throughout the jump. A range of bubble shapes and orientations are present. To maintain consistency with experimental methods, bubbles are assumed spherical, and a closed contour line is deemed a single bubble. The projected area diameter, or the diameter of a sphere with the same projected area as the closed contour, is obtained from $d_{B}=\left(4 A_{B} / \pi\right)^{0.5}$, where $d_{B}$ is the projected area diameter and $A_{B}$ is the $2 \mathrm{D}$ area of the closed contour. The diameters of all recorded bubbles are calculated in this fashion, and a Sauter mean diameter for a given vertical section of the flow is estimated using

$$
d S=j d B 3 j d B 2
$$



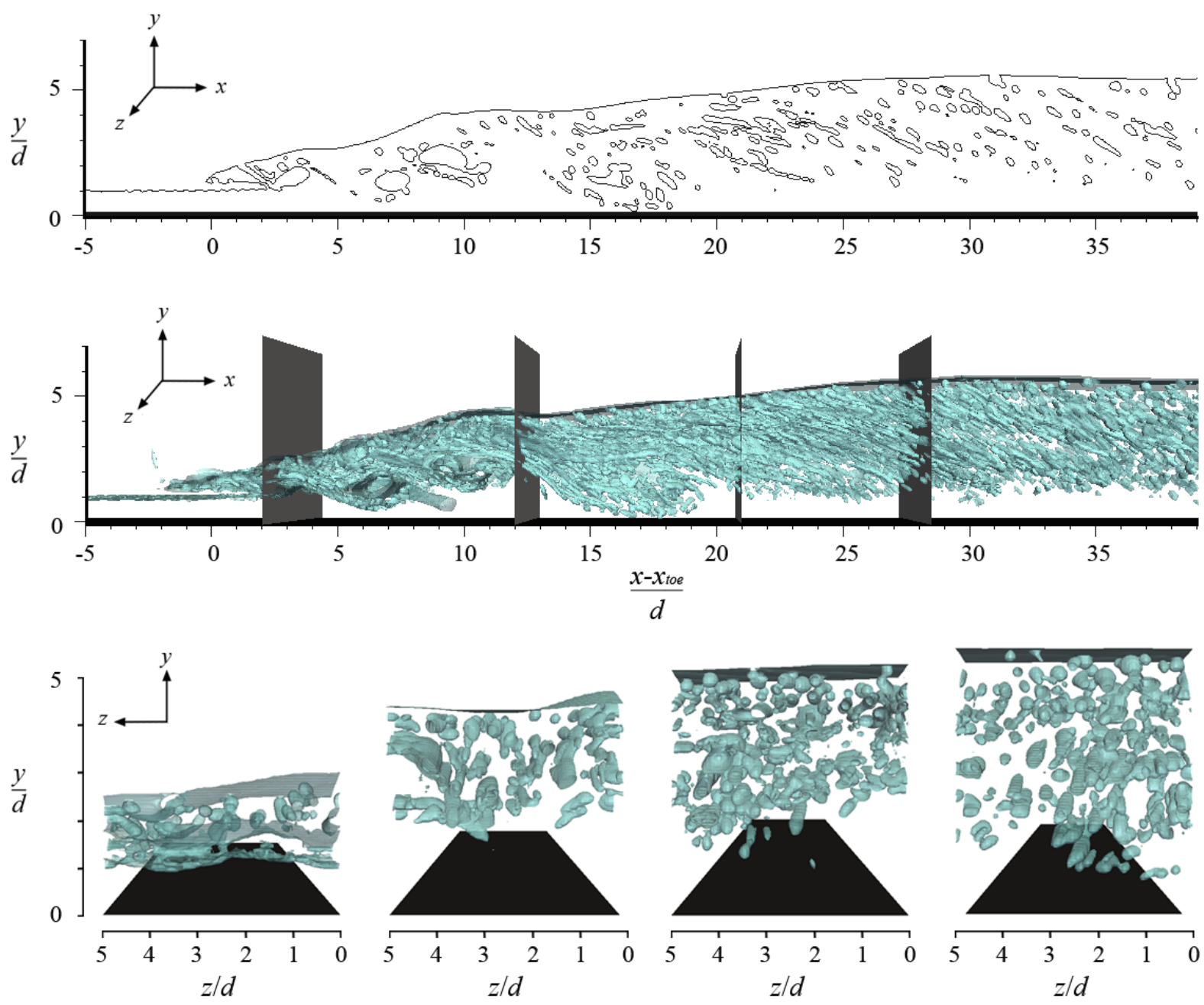

Fig. 11. Instantaneous flow field for $F r=4.82$ 3D. Top: contour of the volume fraction field about a value of $\gamma=$ 0.95 on an $x-y$ plane through the center of the domain $(z=2.5 d)$. Middle: side view, with bubbles represented by 3D isosurfaces about $\gamma=0.95$. Bottom: cross section of the flow field corresponding to each plane in the $3 \mathrm{D}$ view.

The vertical profiles of $d_{s}$ at various downstream locations in the jump are shown in Figs. 12-15. Each plot shows the full range of bubbles observed in the simulation overlaid with simulated and experimental $d_{s}$ as a function of depth normalized by $y_{\varphi 95}$, i.e., the vertical location of $95 \%$ air concentration $(\varphi=0.95)$. The longitudinal measurement locations are the same as those used in void fraction measurements. A contouring algorithm is used to produce an array of $x$ and $y$ coordinates for each distinct closed contour of $\varphi$. The centroid and area of the contour are estimated from these coordinates and each closed contour is assumed to be an independent bubble. A bubble is associated with a particular location if the $x$ coordinate of the centroid of the bubble falls within a $\pm 2 \mathrm{~cm}$ range of the specified horizontal location. 

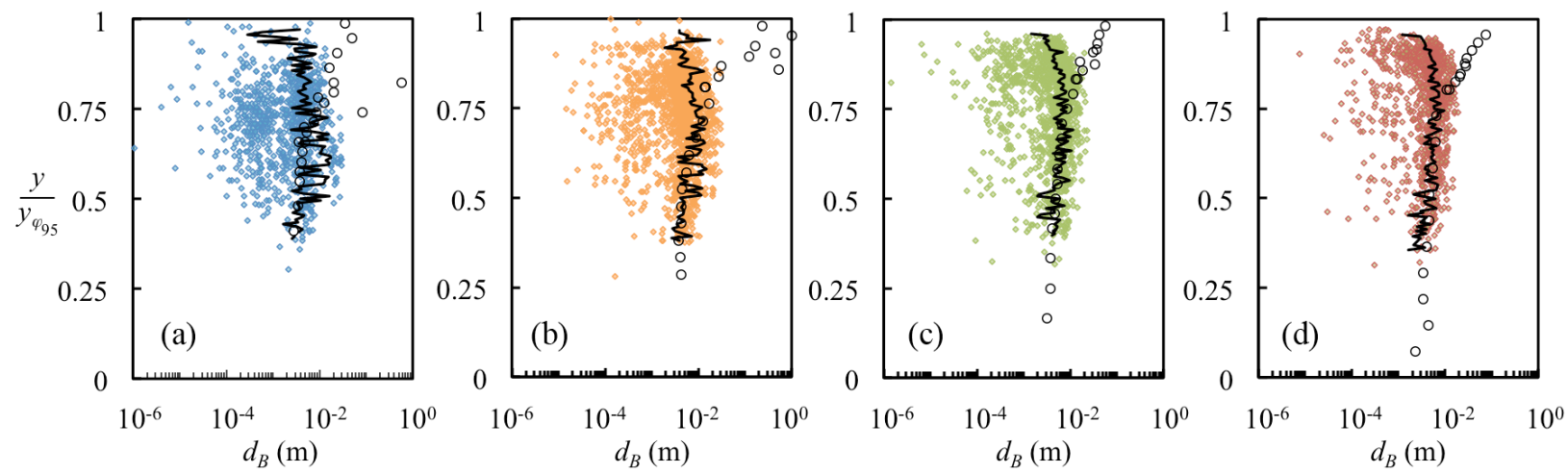

Fig. 12. Bubble diameter for the $F r=2.43$ simulation, plotted against elevation normalized by $y_{\varphi 95}$, for $x-x_{t o e}=$ $2.17 d(\mathrm{a}), 4.35 d(\mathrm{~b}), 6.52 d(\mathrm{c})$, and $8.7 d(\mathrm{~d})$. ' $\mathrm{O}$ ' denotes experimental Sauter mean diameter (Murzyn, 2013); denotes diameter of a single numerical bubble, $d_{B}$; ' ' denotes numerical Sauter mean diameter, $d_{S}$.
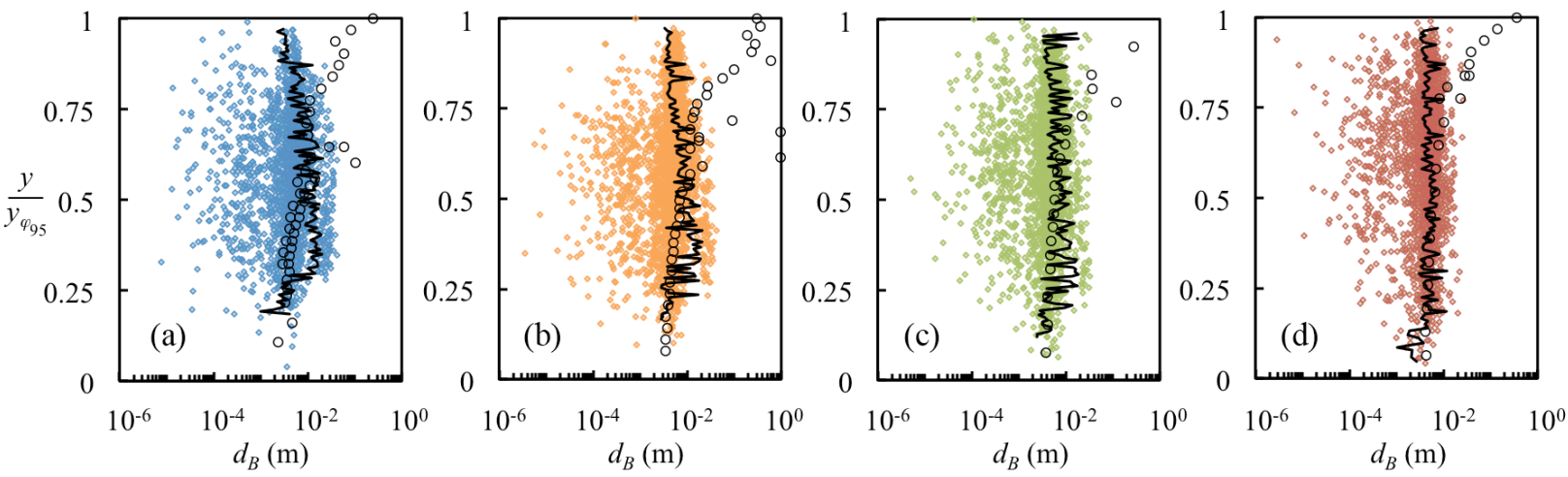

Fig. 13. Sauter mean diameter for the $F r=3.65$ simulation, plotted against elevation normalized by $y_{\varphi 95}$, at $x-x_{t o e}=$ $4.69 d(\mathrm{a}), 7.81 d(\mathrm{~b}), 10.94 d(\mathrm{c})$, and $15.6 d(\mathrm{~d})$. Symbols see Fig. 12.
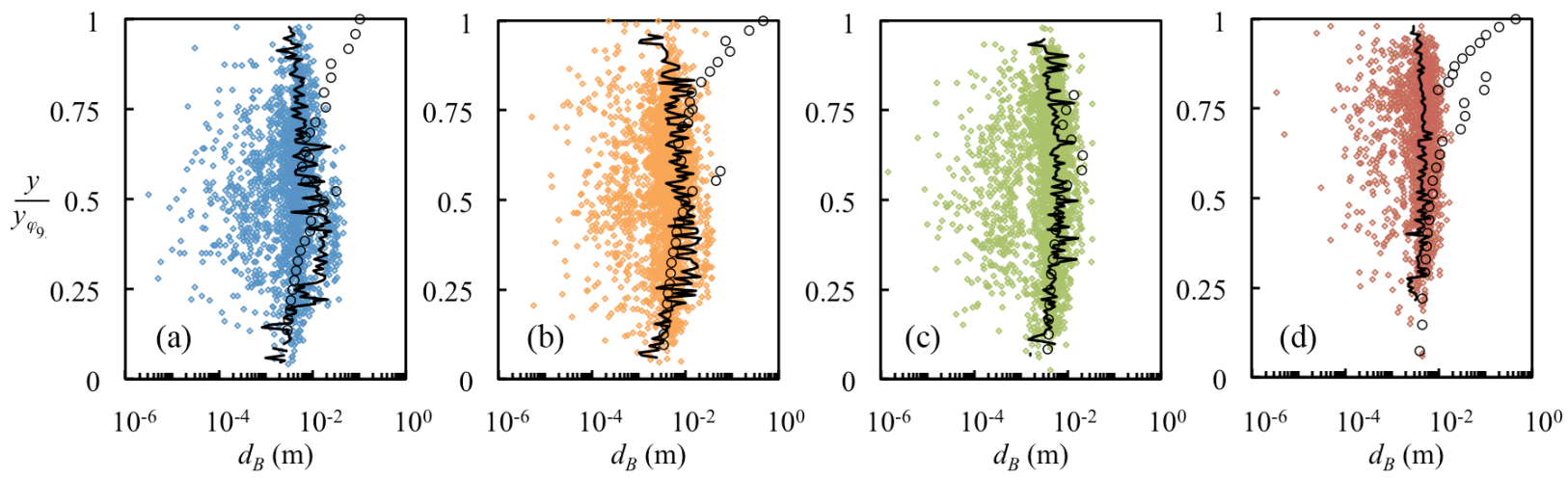

Fig. 14. Sauter mean diameter for the $F r=4.82$ 2D simulation, plotted against elevation normalized by $y_{\varphi 95}$, at $x-x_{\text {toe }}$ $=7.14 d(\mathrm{a}), 11.9 d(\mathrm{~b}), 16.7 d(\mathrm{c})$, and $23.8 d(\mathrm{~d})$. Symbols see Fig. 12 . 

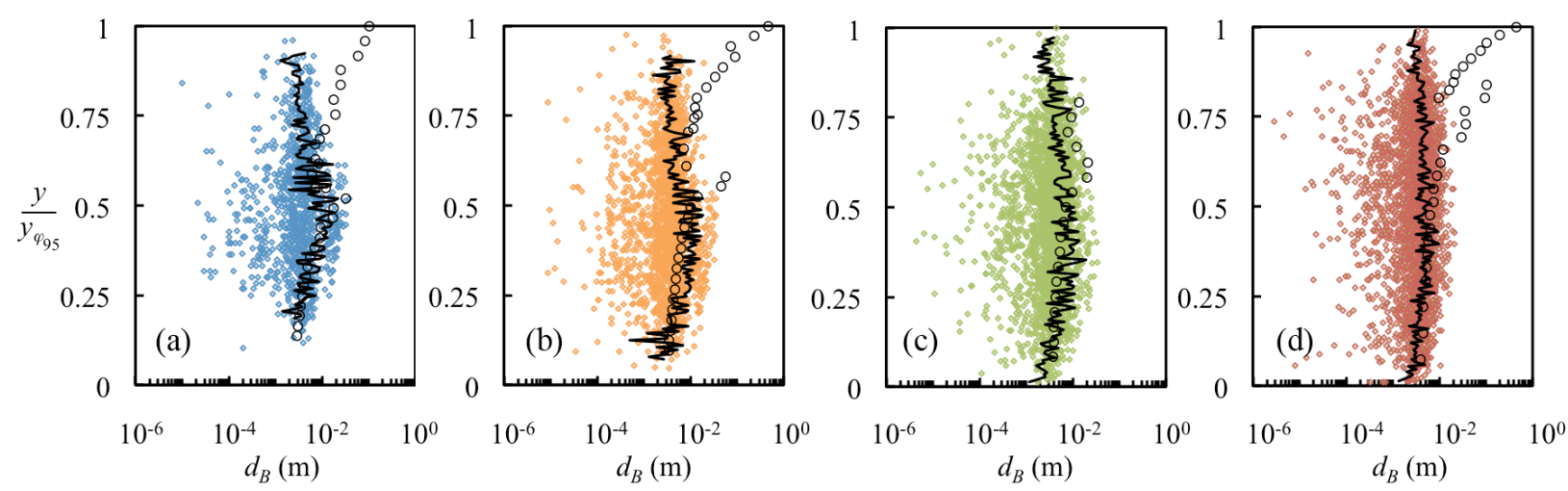

Fig. 15. Sauter mean diameter for $F r=4.823 \mathrm{D}$ simulation, plotted against elevation normalized by $y_{\varphi 95}$, at $x-x_{t o e}=$ 7.14d (a), 11.9d (b), 16.7d (c), and 23.8d (d). Symbols see Fig. 12.

At the first two measurement locations, $d_{S}$ shows an increase from the floor of the jump up through the shear layer $\left(0<y / y_{\varphi 95}<0.5\right)$, qualitatively matching the trend observed in experiments for all $\mathrm{Fr}$ jumps. The 3D simulation exhibits the best agreement, matching the location and size of the maximum experimental $d_{S}$ in the shear layer. Rising above the elevation of entrainment $\left(0.5<y / y_{\varphi 95}<0.75\right), d_{S}$ decreases slightly from the maximum value in the lower shear layer. This trend is more apparent at higher $\mathrm{Fr}$, as was observed in experiments, and correlates closely with a similar trend in void fraction (Figs. 6-8, a and b), indicating the largest bubbles contribute most to maximum air concentrations. Bubble size measurements in this region tend to have a large uncertainty, and can vary up to an order of magnitude for repeated measurements at the same elevation (Murzyn, 2013). Scatter is observed in simulated bubble size as well, though qualitative agreement with experiments is achieved. Both the 2D and 3D simulations produce bubbles throughout the depth of the jump, indicating the advection-diffusion of bubbles is well modeled. For $F r=2.43$ there is minimal downward vertical transport of bubbles after the initial entrainment (Fig. 12a, 12b), while greater Fr jumps show a wider vertical distribution (Fig. 13a, $14 a, 15 a)$.

At the final two measurement locations, bubble size tends to be fairly uniform throughout the depth of the jump, and closely matches experimental $d_{S}$ for all simulated cases. The $2 \mathrm{D}$ simulations tend to underrepresent bubble counts in the lower portion of the jump (Fig. 12c, 12d, 14d). An improvement is seen in the 3D simulation, where the vertical distribution of bubbles spans the full depth of the jump (Fig. $15 \mathrm{c}, 15 \mathrm{~d}$ ), suggesting that the underrepresentation in $2 \mathrm{D}$ simulations is a shortcoming of the numerical approach. The mechanisms responsible for this improvement will be discussed in Section 4.

Sauter mean diameter tends to diverge from experimental estimates near the free surface. In the experimental set-up, optical probes measured the percentage of air at the free surface, which included water surface fluctuations and breaking waves that may not be indicative of distinct bubbles. Our contour approach based on the simulation data ensured that each bubble identified was a distinct bubble with no portion open to the atmosphere. Bubbles open to the atmosphere and breaking waves at the free surface frequently occur in an open channel flow with this level of turbulence. By including their contributions in measurements, an increase in $d_{s}$ near the free surface is expected, i.e., the measurement of bubble sizes are erroneous near the free surface. Murzyn et al. (2005) did not incorporate any bubble size estimates when $\varphi$ was greater than 0.25 for this reason. These results are included herein to emphasize the difficulties associated with obtaining accurate bubble size estimates near a free surface. Simulation results suggest that Sauter mean diameter is close to uniform from the lower depths of the jump up to the free surface, with a slight increase in the shear layer near the toe, where large air pockets are entrained and sheared. Free surface fluctuations contribute to air bubble entrainment; however, we observe the Sauter mean diameter of these bubbles is not substantially larger than those in the turbulent shear region.

Bubble diameter exhibits a log normal distribution at all locations, consistent with the experimental observations of Resch et al. (1974) using hot-wire anemometry, and similar to measurements of air chord 
times of Murzyn and Chanson (2007) using conductivity probes. A range of bubble sizes is represented in the simulations; the smallest simulated bubbles are seen when a single computational cell with $\gamma \approx 0.95$ is surrounded by cells with $\gamma=1$. While the interfacial motion of these bubbles is not physically resolved, the concentration of air contained within them is representative of and equivalent to that of the smallest bubbles in the experiments. Conservation of this air concentration is required to obtain Sauter mean diameter and average void fraction estimates. The simulations did not consider mass transfer, and cannot capture the collapse of bubbles. The mass transfer of volatile compounds such as Oxygen, Nitrogen, and Argon, however, is minimal over the time period of these hydraulic jumps and is not believed to influence bubble size.

With each reduction in grid size the minimum bubble size that can be resolved decreases, and incremental decreases in Sauter mean diameter are expected. Considering the wide range of bubble sizes present in the hydraulic jump, a statistical convergence of bubble diameter may never be achieved. In this work, we have focused on capturing the breakup and transport of bubbles on a Sauter mean diameter scale. By restricting our analysis to bubbles that reside only in the shear layer, Sauter mean diameter shows agreement with experiments throughout the jump (Fig. 16). At nearly all locations, simulated $d_{s}$ is within one standard deviation of experimental values.

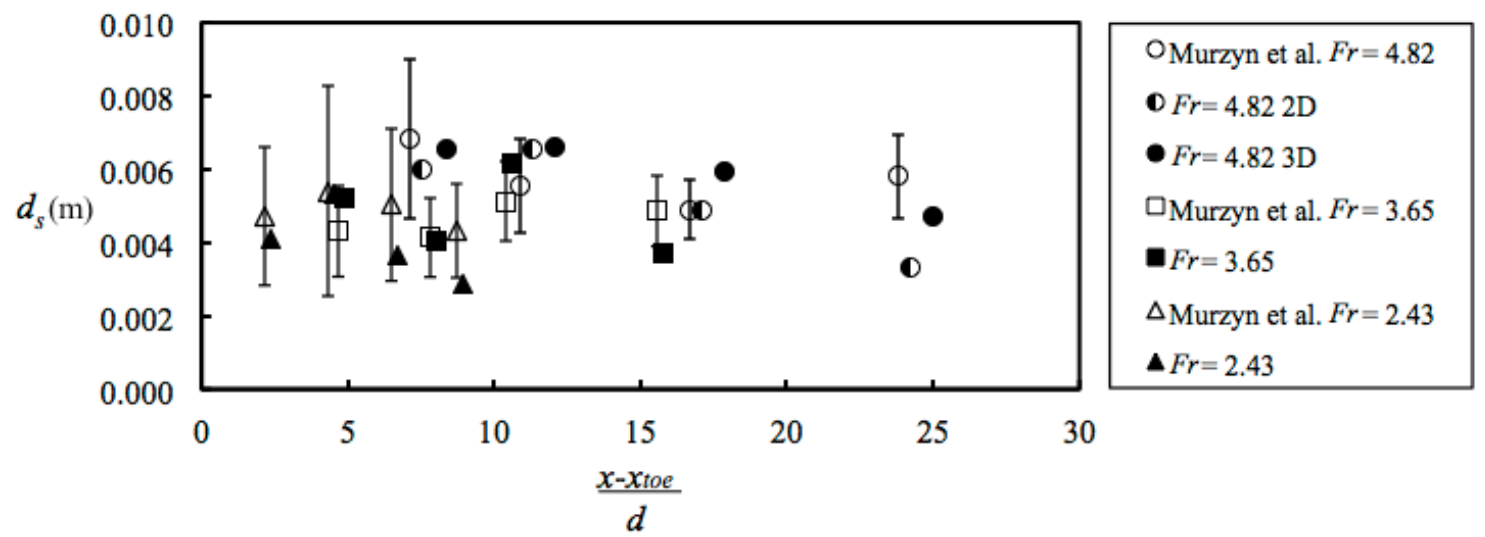

Fig. 16. Depth-averaged Sauter mean diameter within the shear layer. Error bars indicate one standard deviation of experimental values.

A marked improvement towards experimental values of $d_{s}$ is seen as the grid size is reduced. At a grid size of $\Delta x=1.25 \mathrm{~mm}$, the largest bubbles in the shear layer attain a bubble diameter to grid ratio of eight, in agreement with the resolution requirement for bubbles in a VOF method set by Tomiyama et al. (1993). This corresponds with the large improvement in average entrained air seen in Table 1. We conclude that given the computational effort associated with the accuracy of bubble diameter presented herein, a 2D simulation will give an accurate bubble size distribution and depth-averaged void ratio when the largest bubble diameter is represented with eight computational cells, or when four computational cells represent the depth-averaged Sauter mean diameter. If void fraction and total air entrainment are the prime concern, a 2D simulation is accurate to within $10 \%$ of total entrained air. A $3 \mathrm{D}$ simulation improves the vertical bubble distribution and longitudinal bubble transport within the jump. If proper bubble dynamics are more important than average void fraction, a 3D simulation is required, again with at least eight computational cells contained within the largest bubble diameter. 


\section{Vortical structures}

The hydraulic jump is frequently modeled as a $2 \mathrm{D}$ problem when the width to inlet depth ratio is sufficiently large, yet the presence of complex vortical structures and turbulence indicates a 3D phenomenon. These processes are not resolved in 2D simulations, leading to a less accurate prediction of air entrainment and bubble transport. A clear characterization of the additional bubble transport mechanisms resolved in a 3D simulation is required to understand the shortcomings of a 2D simulation. In this section, we visualize the 3D vortical structures associated with primary and secondary bubble transport and describe their importance in mixing and breakup.

Limited experimental evidence suggests the hydraulic jump flow field is similar to a mixing layer (Hoyt and Sellin, 1989; Long et al., 1991; Mossa and Tolve, 1998), yet difficulty in obtaining instantaneous experimental data over space has restricted quantitative analysis. Recent analysis of video images has shown that spanwise vortices are produced at a constant rate that tracks closely with the average oscillation frequency of the toe (Zhang et al., 2012). However, the toe fluctuates only quasiperiodically about a mean value, and frequently exhibits large and small oscillations consecutively in the same direction. It is observed in simulations that these oscillations introduce instabilities of varying intensity and frequency into the upstream jet, which manifest downstream as vortices after a shear-layer roll-up. Under some circumstances the vortices convect downstream at a constant speed, while under other circumstances the vortices may merge, altering their convective velocity. The downstream vorticity signature responds dynamically to a stimulus at the toe, analogous to a forced mixing layer with induced vortex pairing downstream (Ho and Huang, 1982). To explore this behavior further, we next analyze the vorticity structure within the jump during an upstream toe fluctuation, and then during a downstream toe fluctuation.

When the toe is moving upstream, the shear layer appears to experience fairly consistent roll-up. During this regime the roller recirculates an uninterrupted source of bubbly fluid to the toe, and the shear layer instability develops far enough downstream that vortices have limited interactions with the free surface. In Fig. 17 (top), four vortices are present in the shear layer, as visualized by contours of spanwise vorticity. The roller is flattened, meaning the recirculating fluid in the roller cascades towards the toe largely unaffected by the underlying vortical structures, and the free surface exhibits a fairly constant slope starting from the toe and moving downstream. The distance between vortex centers, defined as the location of maximum vorticity within a closed vorticity contour, increases slightly moving downstream, from $4.4 d$ between $\mathrm{A}$ and B, $5.0 d$ between B and C, and $6.25 d$ between $\mathrm{C}$ and $\mathrm{D}$. The vorticity contours exhibit some modulation around larger pockets of air, as was also evidenced in the DNS simulation of a bubble rising through a free shear layer by Taeibi-Rahni et al. (1994). Fig. 17 (bottom) shows an advancement in time of $0.2 \mathrm{~s}$, in which the toe has fluctuated upstream, two new vortices have been generated, and the remaining vortices have convected downstream. The distance between the first four vortex centers increases moving downstream, while the distance between $\mathrm{B}$ and $\mathrm{C}$ decreases as the vortices begin to rotate around each other and pair. This is correlated with a free surface perturbation directly upstream of the merging point. No large perturbations are seen near the toe, consistent with a lack of pairing there. While in a classic mixing layer a lack of vortex pairing reduces the spreading rate (Ho and Huang, 1982), a lack of vortex pairing near the toe of a hydraulic jump flattens the roller, allowing the recirculating flow to spill further and send the toe upstream. 

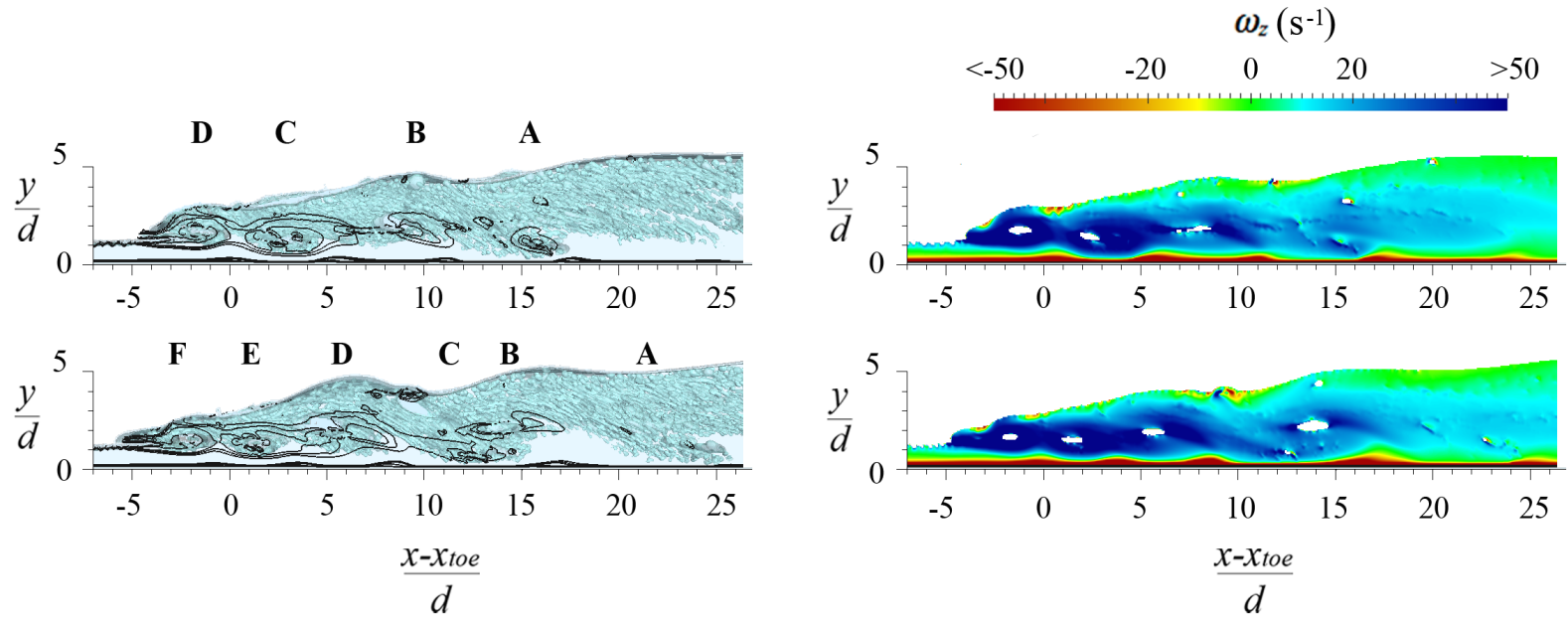

Fig. 17. Visualization of the eddy structure during an upstream fluctuation of the toe. Left: a combination of selected instantaneous vorticity contours $\left(\omega_{\operatorname{mag}}=30,40,50,100,200 \mathrm{~s}^{-1}\right)$ through a 2D $x-y$ plane down the center of the domain. Vorticity maxima inside a vorticity contour are indicated by A through F. Bubbles are represented by a volume fraction isosurface on $\gamma=0.95$. Right: a color contour map of $\omega_{z}$ through a 2D plane down the center of the domain. White areas indicate regions of high void fraction. Top and bottom rows are separated by $0.2 \mathrm{~s}$.

When the toe moves downstream, vortices merging in the shear layer interrupt the reverse flow from the roller creating a supply limited condition. A visualization of vortex pairing during downstream toe fluctuations is shown in Fig. 18. Initially the roller is relatively flat and distinct vortices are present (Fig. 18a). When vortices pair near the toe (Fig. 18b to Fig. 18c, F-E), the free surface is perturbed and rises. This rise in elevation creates a cascade of roller fluid down the upstream side of the elevated free surface. As the impact of the spilling roller deflects the impinging jet downwards a strong roll-up occurs (Fig. 18d, $\mathrm{G}$ ), resulting in vertically offset vortices that begin to rotate around each other (Fig. 18e, H and G). The vorticity contours of $\mathrm{H}-\mathrm{G}$ in Fig. $18 \mathrm{f}$ exhibit a strong similarity to the convective merging of two corotating vortices obtained experimentally (Fig. 1 of Cerretelli and Williamson, 2003). At this point the toe exhibits a fluctuation downstream immediately after vortex merging. Further, more violent disruptions of the free surface result (Fig. 18g), again producing pairs of vertically offset vortices (J, I) that merge and form a roll-braid structure (Fig. 181). The merging of vortices provokes a dynamic response at the free surface that results in greater shear layer instabilities and a fluctuation of the toe downstream. This feedback loop is achieved at some point during the steady roller flow regime (Fig. 17), and appears to intermittently return to that regime after the toe reaches a maximum position downstream.

The convective velocity of vortices, $u_{c}$, is obtained by tracking the vortex centers over time for Fig. 17 and Fig. 18. The result is plotted in Fig. 19. The majority of vortices show a constant convective velocity, with an average of $u_{c} / U_{o}=0.35$ for Fig. 17 and 0.4 for Fig. 18, in line with the value of 0.4 obtained experimentally by Zhang et al (2012) for similar Froude and Reynolds number hydraulic jumps. In the regime where no merging is observed, all vortices within the shear layer travel at a similar speed. At some point, an upstream vortex reaches a critical distance from a downstream vortex and the merging process begins. The mutual influence of each individual vortex results in a counter-clockwise rotation of the vortex pair, forcing the upstream vortex to descend lower to a region of higher horizontal velocity (Fig. 18f, H; Fig. 18k, J). The downstream vortex rotates higher into a lower velocity region, leading to a rise in free surface elevation (Fig. 18f, G; Fig. 18k, I). This interaction occurs quickly, and the resultant merged vortex travels downstream with a nearly constant velocity. 
(a)

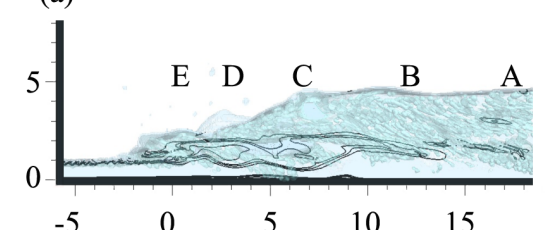

(d)

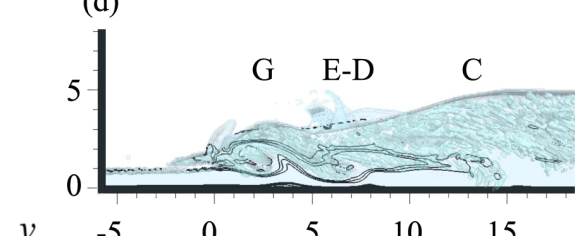

$\frac{y}{d}$
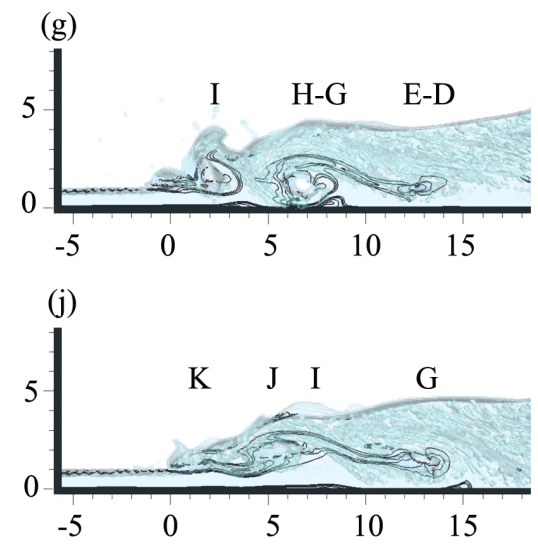

(b)

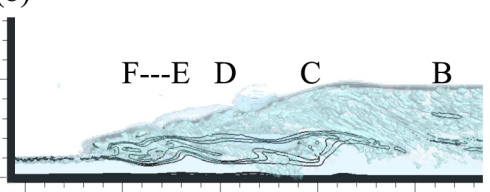

$-5$

(e)
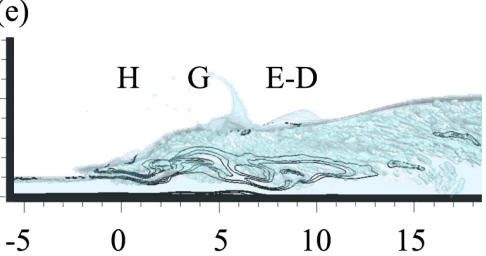

(h)

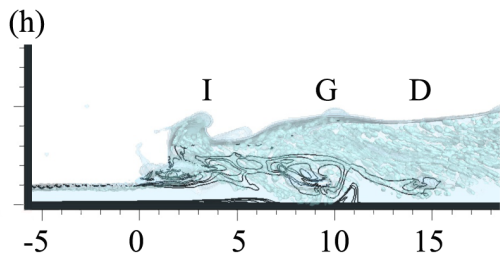

(k)

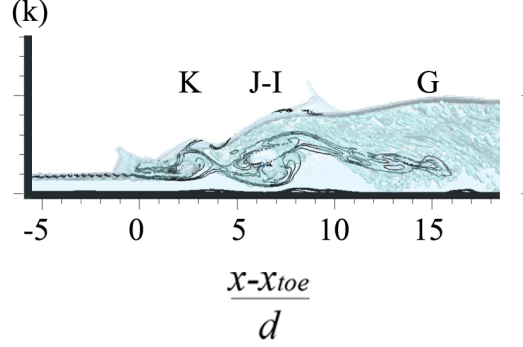

(c)

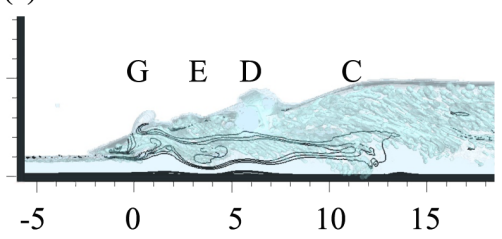

(f)

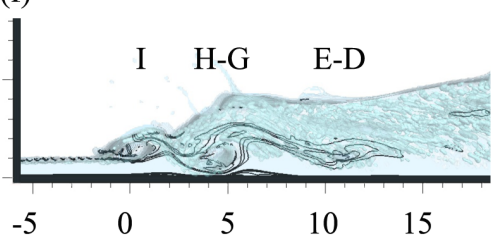

(i)

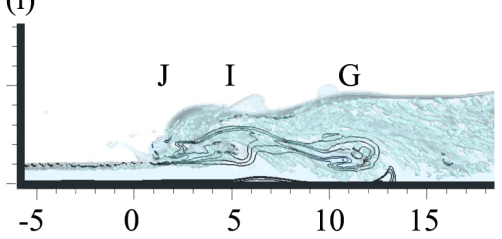

(1)

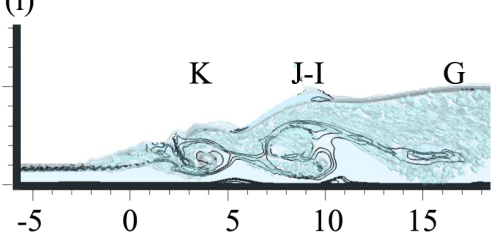

Fig. 18. Visualization of the eddy structure during a downstream fluctuation of the toe. The frame-by-frame representation displays selected instantaneous vorticity contours (black lines) for $\omega_{\text {mag }}=30,40,50,100,200 \mathrm{~s}^{-1}$, through a 2D $x-y$ plane down the center of the domain, and bubbles represented by a volume fraction isosurface on $\gamma$ $=0.95$. Distinct vortices are marked A through $\mathrm{G}$, with the ' - ' indicating merging vortices. For consistency a merged vortex retains the marking of the downstream vortex. The evolution of semi-periodic downstream fluctuations leads to a roll-braid structure (1). Frames are separated by $0.05 \mathrm{~s}$. 

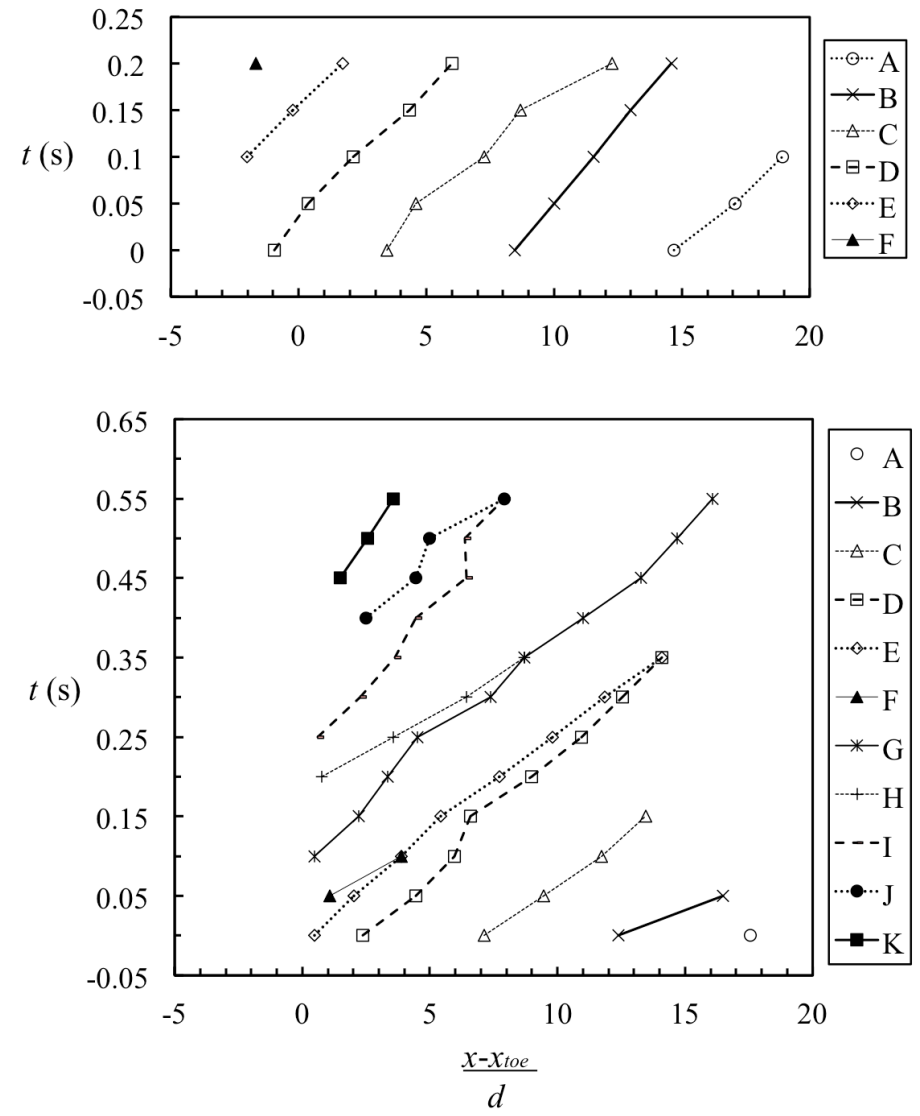

Fig. 19. Vortex center tracked over time. Top: vortices observed from Fig. 17. Bottom: vortices observed from Fig. 18. Letters correspond with vortices from each figure.

Spanwise vortex instabilities generate secondary, streamwise vortices within a mixing layer (Bernal and Roshko, 1986; Hussain, 1986). These streamwise vortices are present in the saddle, or braided region, which is characterized by a relatively small amount of coherent spanwise vorticity, longitudinal vortex stretching, and high shear (Hussain, 1986). Hoyt and Sellin (1989) proposed that a similar braiding process exists in a hydraulic jump, and provided a sketch of a roll-braid structure. Our 3D numerical simulation displayed evidence of a roll-braid structure between two spanwise vortices following the rollup and subsequent pairing and subsequent roll-up of a spanwise vortex (Fig. 18f, Fig. 181). As shown in Fig. 20, counter-rotating streamwise vortex pairs, located in the saddle point between the first and second spanwise vortices downstream from the toe, were observed following the roll-up of merged spanwise vortices (Fig. 20a, b, c). The pairs form near the upper portion of the downstream side of a spanwise vortex, and are oriented diagonally downward towards the upstream side of the neighboring downstream spanwise vortex. Streamwise vorticity is strongest at the upstream side of the pair, in line with the upwelling of fluid from the neighboring upstream spanwise vortex. The interaction of these nearly orthogonal (i.e. spanwise and streamwise) vortices has been shown to produce high 3D turbulence and mixing (Hussain, 1986). Consequently, bubbles transported through this region are consistently observed to experience breakup and are more likely to be transported to lower regions of the flow by the neighboring downstream spanwise vortex. The increased shear provided by streamwise vortices results in improved simulations of void fraction and Sauter mean diameter in 3D compared with 2D, as shown in Sec. 3.

The combined counter-rotating motion between streamwise vortices is observed to contribute to free surface fluctuations (Fig. 20a). The diameter of streamwise vortices is approximately $1 d-2 d$, as is the transverse length of the corresponding free surface fluctuations (Fig. 20a). This is in agreement with the 
transverse free surface integral length scales observed near the toe in experiments with jumps of similar Fr (Chachereau and Chanson, 2011). Longitudinal free surface fluctuations are observed above merged spanwise vortex pairs, with a length of $2 d-3 d$ (Fig. 18g, Fig. 181), also in line with experimental observations. These results suggest the additional upward flux of fluid between streamwise vortex pairs adds a three-dimensional component to free surface fluctuations.

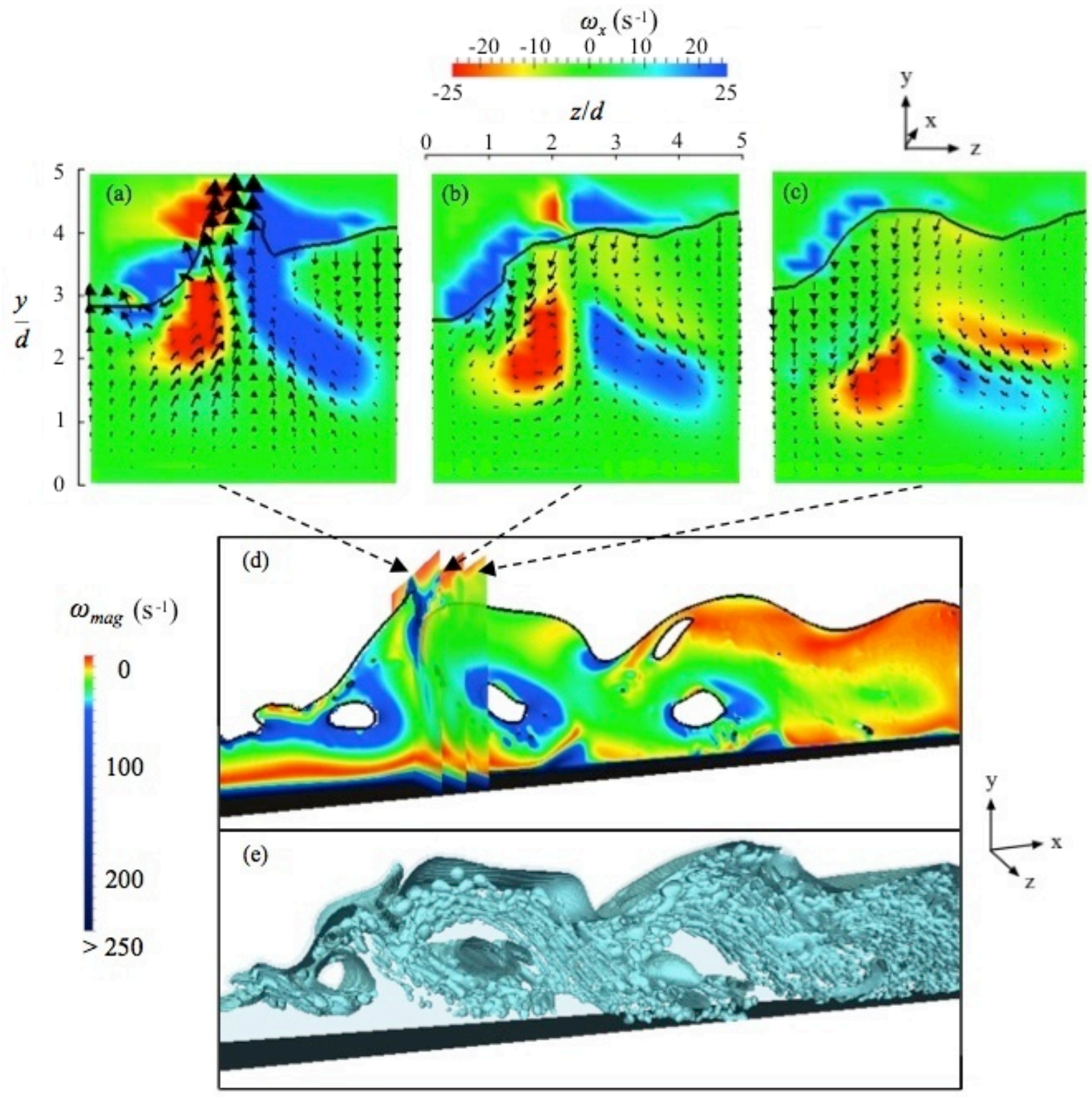

Fig. 20. Counter-rotating streamwise vortices observed between two connected spanwise vortices. (a, b, c) Spanwise, plan views of $\omega_{x}$ separated by $0.5 d$. Arrows represent velocity vectors in the $y$ - $z$ plane, while the solid black line represents the instantaneous free surface. (d) Color contour map of $\omega_{\text {mag }}$ through a 2D plane down the center of the domain. (e) A 3D view of a volume fraction isosurface on $\gamma=0.95$.

A spatially inhomogeneous bubble field is observed in the presence of a braid-roll structure. As large spanwise vortices descend towards the floor, eddies are shed from the high shear region at the wall (Fig. 18g, Fig. 18h). If these eddies have sufficient strength, they are observed to contribute to the breakup of 
the connected roll-braid structure by sending an upwelling of fluid into the saddle (Fig. 18h, Fig. 18i). The fluid in the lower boundary layer is devoid of bubbles, and this injection into the shear layer creates a distinct region with no bubbles. Additionally, spanwise vortices induce some noticeable clustering of bubbles, in that a lack of bubbles is observed immediately downstream of strong spanwise vortices, while the center of each vortex contains a high concentration of bubbles (Fig. 21). This observation is concurrent with experiments, where two regions of high air concentration are observed after a large spill of the roller (Mossa and Tolve, 1998). It is clear from the simulations that each oscillation of the toe gulps pockets of air that travel within rotating spanwise vortices. Smaller bubbles tend to rotate on the exterior of the vortex, where they can be transported to the lower region of the shear layer by the combined action of streamwise vortices and spanwise rotation.
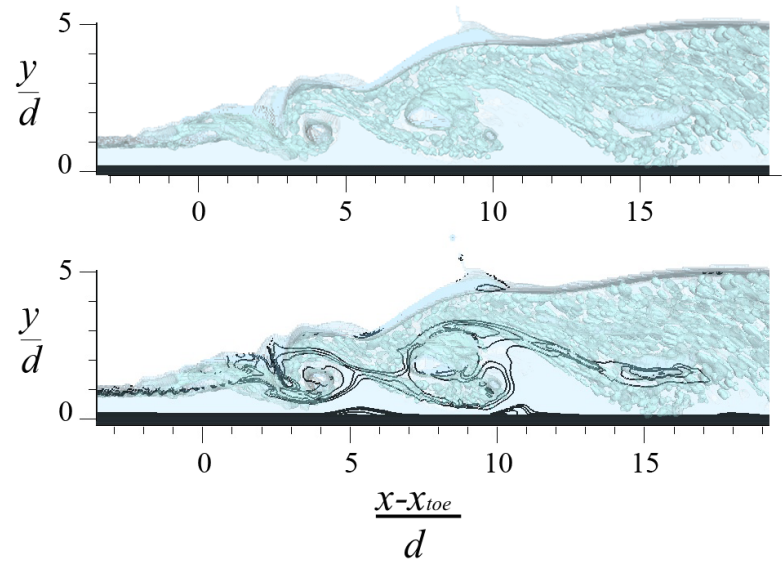

Fig. 21. Selected instantaneous vorticity contours (black lines) for $\omega_{\text {mag }}=30,40,50,100,200 \mathrm{~s}^{-1}$, through a $2 \mathrm{D} x-y$ plane down the center of the domain, and bubbles represented by a volume fraction isosurface on $\gamma=0.95$, during a roll-up of a streamwise vortex (from Fig. 18). Top: spatially inhomogeneous bubble field. Bottom: a lack of bubbles is seen on the lower downstream side of spanwise vortices, below the location of streamwise vortex pairs, and above the region where vorticity is shed from the wall.

In summary, the upstream and downstream oscillations of the toe have a significant effect on vortex dynamics within the jump. The complex interactions of spanwise vortices, wall eddies, and streamwise vortex pairs are not present in 2D simulations. This is believed to have an impact on bubble breakup, coalescence, and transport within the turbulent shear region. As shown in Fig. 8b, the 3D simulation produced a more accurate average void fraction profile within the shear layer. The 3D simulation produced $y_{C \max }$ higher than in the 2D simulation, and was more consistent with experiments. Bubble size was also more accurate in the 3D simulation (Fig. 16). These results suggest improved void fraction and bubble size in a 3D simulation can be attributed to vertical and horizontal bubble transport and bubble breakup due to the combined action of streamwise and spanwise vortical structures. 


\section{Conclusions}

The goal of the present study was to develop a computational framework for the investigation of air entrainment and bubble dynamics in simulated hydraulic jumps. In $2 \mathrm{D}$ and $3 \mathrm{D}$, the RANS equations are solved by interFoam, the VOF method is used to track the interface, and to close the turbulence problem a two equation $k$ - $\varepsilon$ model is used, which is realizable as it considers the restrictions of positivity on normal stresses. Void fraction profiles, velocity profiles, and Sauter mean diameter are validated against experimental data from the literature. The total air entrained by the jump is found to converge to experimental values, while Sauter mean diameter matches experimental estimates within the turbulent shear region. A VOF treatment of the free surface at the proper grid size, sampling rate, and sampling time intrinsically captures air entrainment at a hydraulic jump without the use of an ad hoc air entrainment subgrid model, an improvement over previous simulations reported in the literature. Further, our results confirm that experimental methods for obtaining bubble size estimates near the free surface over-predict Sauter mean diameter.

Our simulations indicate that if the objective of study is to determine the quantity of air entrained by a hydraulic jump, a $2 \mathrm{D}$ simulation will produce results within a $10 \%$ difference of experimental values, without requiring excess computational time, when the diameter of the largest bubbles in the flow is represented by at least eight computational cells. A 3D numerical simulation is recommended if bubble dynamics are considered an important process to model. Improved void fraction profiles and bubble size distributions in a 3D simulation are attributed to improved modeling of bubble transport, and to better resolution of bubble breakup due to the combined action of streamwise and spanwise vortical structures. Improvements come at a cost of two orders of magnitude increase in computational time compared with the $2 \mathrm{D}$ simulation, and are manifest in void fraction profiles immediately downstream from the toe, where 3D turbulence and mixing decrease average bubble size.

Examination of the 3D flow field provides an improved understanding of the flow physics, particularly the behavior of vortical structures and their effect on bubble transport. Spanwise vortices are generated at the confluence of two counter flowing streams, the roller and the impinging jet. Vortex evolution is significantly impacted by the self-forcing mechanism of the recirculating roller, which imparts vorticity into the impinging jet. Unlike a typical mixing layer, the slow stream (i.e. roller) exhibits a dynamic response to the underlying vortex behavior. When vortices convect without merging they have limited interactions with the roller, and steady recirculation moves the jump toe upstream. The toe becomes supply limited and moves downstream when the convective merging of vortices interrupts cascading roller fluid. Vortices merge quickly and maintain a fairly constant velocity downstream. As a consequence of vortex merging, short-lived counter-rotating streamwise vortices are created which combine with spanwise vortices to produce free surface fluctuations. The simulations presented herein reveal flow structures that cannot be visualized experimentally, leading to a better understanding of the flow physics within the hydraulic jump.

\section{Acknowledgements}

This research was supported by funding from the U.S. Department of Energy and its Office of Energy Efficiency and Renewable Energy Water Power Program, through a graduate research fellowship awarded and managed by the Hydro Research Foundation, and from the University of Minnesota through the Department of Civil Engineering's Sommerfeld Fellowship. This work was carried out in part using computing resources at the University of Minnesota Supercomputing Institute. The authors would like to thank Dr. Frédéric Murzyn for providing experimental data. The author also thanks the referees for their comments and suggestions, which were very helpful for the improvement of this paper from the previous version. 


\section{References}

Abbaspour, A., Farsadizadeh, D., Dalir, A.H., Sadraddini, A.A., 2009. Numerical study of hydraulic jumps on corrugated beds using turbulence models. Turkish J. Eng. Env. Sci. 33, 61-72.

Avery, S.T., Novak, P., 1978. Oxygen transfer at hydraulic structures. J. Hydraulics Div. 104, 1521-1540.

Babb, A.F., Aus, H.C., 1981. Measurement of air in flowing water. J. Hydraulics Div. 107, 1615-1630.

Bernal, L., Roshko, A., 1986. Streamwise vortex structure in plane mixing layers. J. Fluid Mech. 170, 499-525.

Boyer, C., Duquenne, A-M., Wild, G., 2002. Measuring techniques in gas-liquid and gas-liquid-solid reactors. Chem. Eng. Sci. 57(16), 3185-3215.

Brackbill, J., Kothe, D.B., Zemach, C., 1992. A continuum method for modeling surface tension. J. Comput. Phys. 100, 335-354.

Brown, G.L., Roshko, A., 1974. On density effects and large structure in turbulent mixing layers. J. Fluid Mech. 64(4), 775816.

Carvalho, R.F., Lemos, C.M., Ramos, C.M., 2008. Numerical computation of the flow in hydraulic jump stilling basins. J. Hydraulic Res. 46(6), 739-752.

Cerretelli, C., Williamson, C., 2003. The physical mechanism for vortex merging. J. Fluid Mech. 475, 41-77.

Chachereau, Y., Chanson, H., 2011. Free-surface fluctuations and turbulence in hydraulic jumps. Exp. Thermal and Fluid Science, 35(6), 896-909.

Chanson, H., 1996. Air bubble entrainment in free surface turbulent flows. Academic Press.

Chanson, H., 2007a. Air bubble entrainment in hydraulic jumps: Physical modeling and scale effects. Proc. $32^{\text {nd }}$ IAHR Congress, Venice, Italy.

Chanson, H., 2007b. Bubbly flow structure in hydraulic jump. European J. Mechanics-B/Fluids 26(3), 367-384.

Chanson, H., 2010. Convective transport of air bubbles in strong hydraulic jumps. Int. J. Multiphase Flow 36(10), 798-814.

Chanson, H., 2013. Hydraulics of aerated flows: qui pro quo? J. Hydraulic Res. 51(3), 223-243.

Chanson, H., Brattberg, T., 2000. Experimental study of the air-water shear flow in a hydraulic jump. Int. J. Multiphase Flow 26(4), 583-607.

Chanson, H., Gualtieri, C., 2008. Similitude and scale effects of air entrainment in hydraulic jumps. J. Hydraulic Res. 46(1), 35-44.

Cheng, X., Chen, X., 2011. Applying Improved Eulerian Model for Simulation of Air-Water Flow in a Hydraulic Jump. Proc. 2011 World Environmental and Water Resources Congress, Palm Springs, California.

Chippada, S., Ramaswamy, B., Wheeler, M.F., 1994. Numerical simulation of hydraulic jump. Int. J. Numer. Meth. Eng. 37, 1381-1397.

Dabiri, D., Gharib, M., 1997. Experimental investigation of the vorticity generation within a spilling water wave. J. Fluid Mech. 330, 113-139.

Deshpande, S.S., Anumolu, L., Trujillo, M.F., 2012. Evaluating the performance of the two-phase flow solver interFoam. Computational Science \& Discovery 5, 014016.

Gonzalez, A., Bombardelli, F., 2005. Two-phase-flow theoretical and numerical models for hydraulic jumps, including air entrainment. Proc. $31^{\text {st }}$ IAHR Congress, Seoul, Korea.

Ho, C.-M., Huang, L.-S., 1982. Subharmonics and vortex merging in mixing layers. J. Fluid Mech. 119, $443-473$.

Hornung, H.G., Willert, C., Turner, S., 1995. The flow field downstream of a hydraulic jump. J. Fluid Mech. 287, $299-316$.

Hoyt, J.W., Sellin, R., 1989. Hydraulic jump as “mixing layer”. J. Hydraulic Eng. 115(12), 1607-1614.

Hussain, A., 1986. Coherent structures and turbulence. J.Fluid Mech. 173, 303-356.

Jasak, H., 1996. Error analysis and estimation for the finite volume method with applications to fluid flows. Ph.D. Thesis, Imperial College of Science, Technology and Medicine, London.

Kim, S.-E., Choudhury, D., Patel, B., 1999. Computations of complex turbulent flows using the commercial code FLUENT. Modeling complex turbulent flows, Springer Netherlands, 259-276.

Krishna, R., van Baten, J.M., 1999. Rise characteristics of gas bubbles in a 2D rectangular column: VOF simulations vs experiments. Int. Comm. in Heat and Mass Transfer 26(7), 965-974.

Lin, C., Hsieh, S., Lin, I., Chang, K., Raikar, R., 2012. Flow property and self similarity in steady hydraulic jumps. Exp. Fluids 53, 1591-1616.

Liu, M., Rajaratnam, N., Zhu, D.Z., 2004. Turbulence structure of hydraulic jumps of low Froude numbers. J. Hydraulic Eng. 130(6), 511-520.

Liu, Q., Drewes, U., 1994. Turbulence characteristics in free and forced hydraulic jumps. J. Hydraulic Res. 32(6), 877-898.

Long, D., Rajaratnam, N., Steffler, P.M., Smy, P.R., 1991. Structure of flow in hydraulic jumps. J. Hydraulic Res. 29(2), 207-218. 
Lubin, P., Glockner, S., Chanson, H., 2009. Numerical simulation of air entrainment and turbulence in a hydraulic jump. Presented at the Colloque SHF Modèles Physiques Hydrauliques: Outils Indispensables du XXIe Siècle, Société Hydrotechnique de France, 109-114.

Ma, J., Oberai, A., Lahey, Jr, R., Drew, D., 2011. Modeling air entrainment and transport in a hydraulic jump using twofluid RANS and DES turbulence models. Heat Mass Transfer 47, 911-919.

Misra, S., Kirby, J., Brocchini, M., Veron, F., Thomas, M., Kambhamettu, C., 2008. The mean and turbulent flow structure of a weak hydraulic jump. Phys. Fluids 20(3), 035106.

Mossa, M., Tolve, U., 1998. Flow visualization in bubbly two-phase hydraulic jump. J. Fluids Eng. 120, 160-165.

Murzyn, F., 2013. Personal communication. Void fraction measurement request.

Murzyn, F., Chanson, H., 2007. Free surface, bubbly flow and turbulence measurement in hydraulic jumps. Research Report No. CH63/07, University of Queensland, Australia.

Murzyn, F., Chanson, H., 2009. Free-surface fluctuations in hydraulic jumps: Experimental observations. Exp. Thermal and Fluid Science 33, 1055-1064.

Murzyn, F., Mouaze, D., Chaplin, J.R., 2005. Optical fibre probe measurements of bubbly flow in hydraulic jumps. Int. J. Multiphase Flow 31, 141-154.

OpenFOAM User Guide, 2012. http://www.openfoam.org/docs/

Pope, S., 2000. Turbulent flows. Cambridge University Press. Chicago.

Qian, Z., Hu, X., Huai, W., Amador, A., 2009. Numerical simulation and analysis of water flow over stepped spillways. Sci. China Ser. E-Technol. Sci. 52, 1958-1965.

Rajaratnam, N., 1962. An experimental study of air entrainment characteristics of the hydraulic jump. J. Inst. Eng. India 42(7), 247-273.

Rajaratnam, N., 1967. Hydraulic jumps. In advances in Hydroscience, Ed. V.T. Chow, Academic Press, New York, USA, Vol. 4, 197-280.

Resch, F.J., Leutheusser, H.J., 1972. Reynolds stress measurements in hydraulic jumps. J. Hydraulic Res. 10(4), 409-430.

Resch, F.J., Leutheusser, H.J., Alemu, S., 1974. Bubbly two-phase flow in hydraulic jump. J. Hydraulics Div. 100 (1), 137149.

Rodríguez-Rodríguez, J., Marugán-Cruz, C., Aliseda, A., Lasheras, J.C., 2011. Dynamics of large turbulent structures in a steady breaker. Exp. Thermal and Fluid Science 35, 301-310.

Rusche, H., 2002. Computational fluid dynamics of dispersed two-phase flows at high phase fractions. Ph.D. Thesis, Imperial College of Science, Technology and Medicine, London.

Shih, T-H., Liou, W.W., Shabbir, A., Yang, Z., Zhu, J., 1995. A new k- $\epsilon$ eddy viscosity model for high Reynolds number turbulent flows. Computers \& Fluids 24, 227-238.

Souders, D.T., Hirt, C., 2004. Modeling entrainment of air at turbulent free surfaces. Proc. World Water and Environmental Resources Congress, pp. 1-10.

Straub, L.G., A. G. Anderson, 1958. Experiments on self-aerated flow in open channels. J. Hydraulics Div. ASCE, 84(7), 135 .

Taeibi-Rahni, M., Loth, E., Tryggvason, G., 1994. Flow modulation of a planar free shear layer with large bubbles - direct numerical simulations. Int. J. Multiphase Flow 20(6), 1109-1128.

Tomiyama, A., Sou, A., Minagawa, H., Sakaguchi, T., 1993. Numerical analysis of a single bubble by VOF method. JSME Int. J. Ser. B. 36, 51-56.

Waniewski, T.A., Hunter, C., Brennen, C.E., 2001. Bubble measurements downstream of hydraulic jumps. Int. J. Multiphase Flow 27(7), 1271-1284.

Wilhelms, S.C., Gulliver, J.S., 2005. Gas transfer, cavitation and bulking in self-aerated spillway flow. J. Hydraulic Res. 43(5), 532-539.

Zhang, G., Wang, H., Chanson, H., 2012. Turbulence and aeration in hydraulic jumps: free-surface fluctuation and integral turbulent scale measurements. Environ Fluid Mech. 13(2), 189-204.

Zhao, Q., Misra, S.K., Svendsen, I.A., Kirby, J.T. 2004. Numerical study of a turbulent hydraulic jump. Proc. $17^{\text {th }}$ Eng. Mech. Div. Conf. ASCE, Newark, USA.

Zhu, J., Shih, T-H., 1994. Computation of confined coflow jets with 3 turbulence models. Int. J. Numer. Methods Fluids 19, 939-956.

Zidouni, K.F., Bousbia, S.A., Mataoui, A., 2010. Assessment of three turbulence model performances in predicting water jet flow plunging into a liquid pool. Nuclear Technology and Radiation Protection 25(1), 13-22. 